\title{
Article \\ On the First-Passage Time Problem for a Feller-Type Diffusion Process
}

\author{
Virginia Giorno ${ }^{*}+(\mathbb{D})$ and Amelia G. Nobile ${ }^{+}(\mathbb{D}$ \\ Dipartimento di Informatica, Università degli Studi di Salerno, Via Giovanni Paolo II n. 132, \\ 84084 Fisciano, Salerno, Italy; nobile@unisa.it \\ * Correspondence: giorno@unisa.it \\ $\uparrow$ These authors contributed equally to this work.
}

Citation: Giorno, V.; Nobile, A.G. On the First-Passage Time Problem for a Feller-Type Diffusion Process. Mathematics 2021, 9, 2470. https:/ / doi.org/10.3390/math9192470

Academic Editor: Palle E. T. Jorgensen

Received: 2 September 2021

Accepted: 26 September 2021

Published: 3 October 2021

Publisher's Note: MDPI stays neutral with regard to jurisdictional claims in published maps and institutional affiliations.

Copyright: (c) 2021 by the authors. Licensee MDPI, Basel, Switzerland. This article is an open access article distributed under the terms and conditions of the Creative Commons Attribution (CC BY) license (https:// creativecommons.org/licenses/by/ $4.0 /)$.

\begin{abstract}
We consider the first-passage time problem for the Feller-type diffusion process, having infinitesimal drift $B_{1}(x, t)=\alpha(t) x+\beta(t)$ and infinitesimal variance $B_{2}(x, t)=2 r(t) x$, defined in the space state $[0,+\infty)$, with $\alpha(t) \in \mathbb{R}, \beta(t)>0, r(t)>0$ continuous functions. For the timehomogeneous case, some relations between the first-passage time densities of the Feller process and of the Wiener and the Ornstein-Uhlenbeck processes are discussed. The asymptotic behavior of the first-passage time density through a time-dependent boundary is analyzed for an asymptotically constant boundary and for an asymptotically periodic boundary. Furthermore, when $\beta(t)=\xi r(t)$, with $\xi>0$, we discuss the asymptotic behavior of the first-passage density and we obtain some closed-form results for special time-varying boundaries.
\end{abstract}

Keywords: first-passage time densities; Laplace transforms; Wiener process; Ornstein-Uhlenbeck process; first-passage time moments; asymptotic behaviors

MSC: 60J60; 60J70; 44A10

\section{Introduction}

Diffusion processes are often used to describe the development of dynamic systems in a broad variety of scientific disciplines, including physics, biology, population dynamics, neurology, finance, and queueing. There is much interest in analyzing the "first-passage time" (FPT) issue in various situations. This entails determining the probability distribution of a random variable that describes the moment at which a process, beginning from a fixed initial state, reaches a defined boundary or threshold for the first time, which may also be time-varying. Unfortunately, closed-form solutions for the FPT densities are only accessible in a limited number of instances, leaving the more difficult job of determining the FPT densities through time-dependent boundaries.

Some general methods to solve FPT problems are based on:

1. Analytical methods to determine the Laplace transform of FPT probability density function (pdf) and its moments for time-homogeneous diffusion process and constant boundaries (cf., for instance, Darling and Siegert [1], Blake and Lindsey [2], Giorno et al. [3]);

2. Symmetry properties of transition density to obtain closed-form results on the FPT densities through time-dependent boundaries and other related functions (cf., for instance, Di Crescenzo et al. [4]);

3. Construction of FPT pdf by making use of certain transformations among diffusion processes (cf., for instance, Gutiérrez et al. [5], Di Crescenzo et al. [6], Giorno and Nobile [7]);

4. Formulation of integral equations for the FPT density (cf., for instance, Buonocore et al. [8], Gutiérrez et al. [9], Di Nardo et al [10]); 
5. Analysis of the asymptotic behavior of FPT pdf for large boundary or large times (cf., for instance, Nobile et al. [11,12])

6. Efficient numerical algorithms and simulation procedures to estimate FPT pdf's (cf., for instance, Herrmann and Zucca [13], Giraudo et al. [14], Taillefumier and Magnasco [15], Giorno and Nobile [16], Naouara and Trabelsi [17]).

In the present paper, we focus on the FPT problem for the Feller-type diffusion process. Let $\left\{X(t), t \geq t_{0}\right\}, t_{0} \geq 0$, be a time-inhomogeneous Feller-type diffusion process, defined in the state space $[0,+\infty)$, which satisfies the following stochastic differential equation:

$$
d X(t)=[\alpha(t) X(t)+\beta(t)] d t+\sqrt{2 r(t) X(t)} d W(t), \quad X\left(t_{0}\right)=x_{0},
$$

where $W(t)$ is a standard Wiener process. Hence, the infinitesimal drift and infinitesimal variance of $X(t)$ are

$$
B_{1}(x, t)=\alpha(t) x+\beta(t), \quad B_{2}(x, t)=2 r(t) x
$$

and we assume that $\alpha(t) \in \mathbb{R}, \beta(t)>0, r(t)>0$ are continuous functions for all $t \geq t_{0}$.

The Feller diffusion process plays a relevant role in different fields: in mathematical biology to model the growth of a population (cf. Feller [18], Lavigne and Roques [19], Masoliver [20], Pugliese and Milner [21]), in queueing systems to describe the number of customers in a queue (cf. Di Crescenzo and Nobile [22]), in neurobiology to analyze the input-output behavior of single neurons (see, for instance, Giorno et al. [23], Buonocore et al. [24], Ditlevsen and Lánský [25], Lánský et al. [26], Nobile and Pirozzi [27], D'Onofrio et al. [28]), in mathematical finance to model interest rates and stochastic volatility (see Cox et al. [29], Tian and Zhang [30], Maghsoodi [31], Peng and Schellhorn [32]). In population dynamics, the Feller-type diffusion process arises as a continuous approximation of a linear birth-death process with immigration (cf., for instance, Giorno and Nobile [33]). The Feller process has the advantage of having a state space bounded from below, a property that in the neuronal models allows the inclusion of the effect of reversal hyperpolarization potential. In this context, the statistical estimation of parameters of the Feller process starting from observations of its first-passage times plays a relevant role (cf., for instance, Ditlevsen and Lánský [25], Ditlevsen and Ditlevsen [34]). The study of the Feller process is also interesting in chemical reaction dynamics (cf., for instance, [35]).

For the Feller-type diffusion process $X(t)$, we assume that the total probability mass is conserved in $(0,+\infty)$ and we denote by $f\left(x, t \mid x_{0}, t_{0}\right)=\partial P\left\{X(t) \leq x \mid X\left(t_{0}\right)=x_{0}\right\} / \partial x$ the transition pdf of $X(t)$ in the presence of a zero-flux condition in the zero state (cf., for instance, Giorno and Nobile [33]). Moreover, for the process $X(t)$, we consider the random variable

$$
\mathcal{T}\left(x_{0}, t_{0}\right)= \begin{cases}\inf _{t \geq t_{0}}\{t: X(t) \geq S(t)\}, & X\left(t_{0}\right)=x_{0}<S\left(t_{0}\right), \\ \inf _{t \geq t_{0}}\{t: X(t) \leq S(t)\}, & X\left(t_{0}\right)=x_{0}>S\left(t_{0}\right),\end{cases}
$$

which denotes the FPT of $X(t)$ from $X\left(t_{0}\right)=x_{0}$ to the continuous boundary $S(t)$. The FPT pdf $g\left[S(t), t \mid x_{0}, t_{0}\right]=\partial P\left(\mathcal{T}\left(x_{0}, t_{0}\right) \leq t\right) / \partial t$ satisfies the first-kind Volterra integral equation (cf., for instance, Fortet [36]):

$$
\begin{aligned}
f\left(x, t \mid x_{0}, t_{0}\right)=\int_{t_{0}}^{t} g\left[S(u), u \mid x_{0}, t_{0}\right] f[x, t \mid S(u), u] d u \\
\\
\quad\left[x_{0}<S\left(t_{0}\right), x \geq S(t)\right] \text { or }\left[x_{0}>S\left(t_{0}\right), x \leq S(t)\right] .
\end{aligned}
$$

The renewal Equation (3) expresses that any sample path that reaches $x \geq S(t)$ $[x \leq S(t)]$, after starting from $x_{0}<S\left(t_{0}\right)\left[x_{0}>S\left(t_{0}\right)\right]$ at time $t_{0}$, must necessarily cross $S(u)$ for the first time at some intermediate instant $u \in\left(t_{0}, t\right)$. Research on the FPT problem 
for the Feller diffusion process has been carried out by Giorno et al. [37], Linetsky [38], Masoliver and Perelló [39], Masoliver [40], Chou and Lin [41], Di Nardo and D'Onofrio [42], Giorno and Nobile [43]).

The paper is structured as follows. In Section 2, we consider the time-homogeneous Feller process with a zero-flux condition in the zero state. For this process, we analyze the FPT problem through a constant boundary $S$ starting from the initial state $x_{0}$ by determining the Laplace transform of the FPT density and the ultimate FPT probability in the following cases: (a) $x_{0}>S \geq 0$ and (b) $0 \leq x_{0}<S$. In particular, a closed-form expression for the FPT pdf through the zero state is given. Moreover, some connections between the FPT densities of the Feller process and the Wiener and Ornstein-Uhlenbeck processes are investigated. In Section 3, making use of the iterative Siegert formula, the first three FPT moments are obtained and analyzed. In Section 4, we study the asymptotic behavior of the FPT density when the time-varying boundary $S(t)$ moves away from the starting point $x_{0}$ for large time by distinguishing two cases: $S(t)$ is an asymptotically constant boundary and $S(t)$ is an asymptotically periodic boundary.

Section 5 is dedicated to the time-inhomogeneous Feller process in the proportional case. Specifically, we assume that $\alpha(t)$ is a real function, $r(t)>0$ and $\beta(t)=\xi r(t)$, with $\xi>0$. For this case, we determine the closed-form expression of the FPT density through the zero state. Furthermore, for $\xi=1 / 2$ and $\xi=3 / 2$, we obtain the FPT density through a specific time-varying boundary and the related ultimate FPT probability. Finally, in Section 6 , an asymptotic exponential approximation is derived for asymptotically constant boundaries.

Various numerical computations are performed both for the time-homogeneous Feller process and for the time-inhomogeneous Feller-type process to analyze the role of the parameters.

\section{FPT Problem for a Time-Homogeneous Feller Process}

We consider the time-homogeneous Feller process $X(t)$ with drift $B_{1}(x)=\alpha x+\beta$ and infinitesimal variance $B_{2}(x)=2 r x$, defined in the state space $[0,+\infty)$. As proved by Feller [44], the state $x=0$ is an exit boundary for $\beta \leq 0$, a regular boundary for $0<\beta<r$ and an entrance boundary for $\beta \geq r$. The scale function and the speed density of $X(t)$ are (cf. Karlin and Taylor [45]):

$$
\begin{aligned}
& h(x)=\exp \left\{-2 \int^{x} \frac{B_{1}(z)}{B_{2}(z)} d z\right\}=x^{-\beta / r} \exp \left\{-\frac{\alpha x}{r}\right\}, \\
& s(x)=\frac{2}{B_{2}(x) h(x)}=\frac{x^{\beta / r-1}}{r} \exp \left\{\frac{\alpha x}{r}\right\},
\end{aligned}
$$

respectively. In this section, we assume that $\beta>0$ and suppose that a zero-flux condition is placed in the zero state.

\subsection{Transition Density}

When $\alpha \in \mathbb{R}, \beta>0$ and $r>0$, imposing a zero-flux condition in the zero state, the transition pdf of $X(t)$ can be explicitly obtained (cf., for instance, Giorno et al. [37], Sacerdote [46]). Indeed, when $\alpha=0, \beta>0$ and $r>0$, the transition pdf is:

$$
f\left(x, t \mid x_{0}, t_{0}\right)=\left\{\begin{array}{cc}
\frac{1}{x \Gamma(\beta / r)}\left[\frac{x}{r\left(t-t_{0}\right)}\right]^{\beta / r} \exp \left\{-\frac{x}{r\left(t-t_{0}\right)}\right\}, & x_{0}=0, \\
\frac{1}{r\left(t-t_{0}\right)}\left(\frac{x}{x_{0}}\right)^{(\beta-r) /(2 r)} \exp \left\{-\frac{x_{0}+x}{r\left(t-t_{0}\right)}\right\} & \\
\times I_{\beta / r-1}\left[\frac{\left.2 \sqrt{x x_{0}}\right]}{r\left(t-t_{0}\right)}\right], & x_{0}>0,
\end{array}\right.
$$


whereas if $\alpha \neq 0, \beta>0$ and $r>0$, one obtains:

$$
f\left(x, t \mid x_{0}, t_{0}\right)= \begin{cases}\frac{1}{x \Gamma(\beta / r)}\left[\frac{\alpha x}{r\left(e^{\alpha\left(t-t_{0}\right)}-1\right)}\right]^{\beta / r} \exp \left\{-\frac{\alpha x}{r\left(e^{\alpha\left(t-t_{0}\right)}-1\right)}\right\}, & x_{0}=0, \\ \frac{\alpha}{r\left(e^{\alpha\left(t-t_{0}\right)}-1\right)}\left[\frac{x e^{-\alpha\left(t-t_{0}\right)}}{x_{0}}\right]^{(\beta-r) /(2 r)} \exp \left\{-\frac{\alpha\left[x+x_{0} e^{\alpha\left(t-t_{0}\right)}\right]}{r\left(e^{\alpha\left(t-t_{0}\right)}-1\right)}\right\} & \\ \times I_{\beta / r-1}\left[\frac{2 \alpha \sqrt{x x_{0} e^{\alpha\left(t-t_{0}\right)}}}{r\left(e^{\alpha\left(t-t_{0}\right)}-1\right)}\right], & x_{0}>0,\end{cases}
$$

where

$$
I_{v}(z)=\sum_{k=0}^{+\infty} \frac{1}{k ! \Gamma(v+k+1)}\left(\frac{z}{2}\right)^{2 k+v}, \quad v \in \mathbb{R}
$$

denotes the modified Bessel function of the first kind and $\Gamma(\xi)$ is Eulero's gamma function. Here and elsewhere, whenever the multiple-valued functions such as $\left(\frac{z}{2}\right)^{2 k+v}$ appear, they are assumed to be taken as their principal branches. We note that the transition pdf $f\left(x, t \mid x_{0}, t_{0}\right)$ in (5) and (6) satisfies the following relation:

$$
f\left(x, t \mid x_{0}, t_{0}\right)=\left(\frac{x}{x_{0}}\right)^{\beta / r-1} \exp \left\{\frac{\alpha\left(x-x_{0}\right)}{r}\right\} f\left(x_{0}, t \mid x, t_{0}\right), \quad x_{0}>0, x>0 .
$$

Moreover, when $\alpha<0, \beta>0$ and $r>0$, the time-homogeneous Feller process allows a steady-state density:

$$
W(x)=\lim _{t \rightarrow+\infty} f\left(x, t \mid x_{0}, t_{0}\right)=\frac{1}{x \Gamma(\beta / r)}\left(\frac{|\alpha| x}{r}\right)^{\beta / r} \exp \left\{-\frac{|\alpha| x}{r}\right\}, \quad x>0,
$$

which is a gamma density of parameters $\beta / r$ and $r /|\alpha|$. In the sequel, we denote by

$$
q_{\lambda}\left(x \mid x_{0}\right)=\int_{0}^{+\infty} e^{-\lambda t} q\left(x, t \mid x_{0}\right) d t, \quad \lambda>0
$$

the Laplace transform (LT) of the function $q\left(x, t \mid x_{0}\right) \equiv q\left(x, t \mid x_{0}, 0\right)$.

\subsection{Laplace Transform of the Transition Density}

By performing the LT to (5) and (6), for $0 \leq x_{0}<x$ one has (cf. Giorno et al. [37], Chou and Lin [41]):

$$
f_{\lambda}\left(x \mid x_{0}\right)= \begin{cases}\frac{e^{-|\alpha| x / r} x^{\beta / r-1}}{|\alpha| \Gamma(\beta / r)}\left(\frac{|\alpha|}{r}\right)^{\beta / r} \Gamma\left(\frac{\lambda}{|\alpha|}\right) \Psi\left(\frac{\lambda}{|\alpha|}, \frac{\beta}{r} ; \frac{|\alpha| x}{r}\right) \Phi\left(\frac{\lambda}{|\alpha|}, \frac{\beta}{r} ; \frac{|\alpha| x_{0}}{r}\right), & \alpha<0, \\ \frac{2}{r}\left(\frac{x}{x_{0}}\right)^{\beta /(2 r)-1 / 2} K_{\beta / r-1}\left(2 \sqrt{\frac{\lambda x}{r}}\right) I_{\beta / r-1}\left(2 \sqrt{\frac{\lambda x_{0}}{r}}\right), & \alpha=0, \\ \frac{e^{-\alpha x_{0} / r} x^{\beta / r-1}}{\alpha \Gamma(\beta / r)}\left(\frac{\alpha}{r}\right)^{\beta / r} \Gamma\left(\frac{\lambda}{\alpha}+\frac{\beta}{r}\right) \Psi\left(\frac{\lambda}{\alpha}+\frac{\beta}{r}, \frac{\beta}{r} ; \frac{\alpha x}{r}\right) \Phi\left(\frac{\lambda}{\alpha}+\frac{\beta}{r}, \frac{\beta}{r} ; \frac{\alpha x_{0}}{r}\right), & \alpha>0,\end{cases}
$$

where

$$
K_{v}(x)=\frac{\pi}{2} \frac{I_{-v}(x)-I_{v}(x)}{\sin (v \pi)}
$$


denotes the modified Bessel function of the second kind (cf. Gradshteyn and Ryzhik [47], p. 928, no. 8.485) and

$$
\begin{aligned}
& \Phi(a, c ; x)=1+\sum_{n=1}^{+\infty} \frac{(a)_{n}}{(c)_{n}} \frac{x^{n}}{n !}, \\
& \Psi(a, c ; x)=\frac{\Gamma(1-c)}{\Gamma(a-c+1)} \Phi(a, c ; x)+\frac{\Gamma(c-1)}{\Gamma(a)} x^{1-c} \Phi(a-c+1,2-c ; x),
\end{aligned}
$$

are the Kummer's functions of the first and second kinds, respectively (cf. Gradshteyn and Ryzhik [47], p. 1023, no. 9.210.1 and no. 9.210.2). Kummer's functions satisfy the following relations (cf. Tricomi [48]):

$$
\Phi(a, c ; x)=e^{x} \Phi(c-a, c ;-x), \quad \Phi(a, a ; x)=e^{x}
$$

and

$$
\Psi(a, c ; x)=x^{1-c} \Psi(a-c+1,2-c ; x), \quad \Psi(0, c ; x)=1, \quad \Psi(c, c ; x)=e^{x} \Gamma(1-c, x),
$$

where

$$
\Gamma(a, x)=\int_{x}^{+\infty} e^{-t} t^{a-1} d t
$$

denotes the incomplete gamma function. By performing the Laplace transform to both sides of (8), the following result is obtained:

$$
f_{\lambda}\left(x \mid x_{0}\right)=\left(\frac{x}{x_{0}}\right)^{\beta / r-1} \exp \left\{\frac{\alpha\left(x-x_{0}\right)}{r}\right\} f_{\lambda}\left(x_{0} \mid x\right), \quad x_{0}>0, x>0 .
$$

\subsection{Laplace Transform of the FPT Density}

An analytic approach to analyze the FPT problem through a non-negative constant boundary $S(t)=S$ is based on the Laplace transform. Indeed, from (3), one has:

$$
g_{\lambda}\left(x \mid x_{0}\right)=\frac{f_{\lambda}\left(x \mid x_{0}\right)}{f_{\lambda}(x \mid S)}, \quad\left[x_{0}<S \leq x\right] \text { or }\left[x \leq S<x_{0}\right]
$$

so that the LT of the FPT pdf $g\left(S, t \mid x_{0}\right)$ can be evaluated by knowing the LT of the transition $\operatorname{pdf} f\left(S, t \mid x_{0}\right)$.

To determine $g_{\lambda}\left(S \mid x_{0}\right)$ via (17), we consider the following cases: (a) $x_{0}>S \geq 0$ and (b) $0 \leq x_{0}<S$.

(a) FPT downwards for the time-homogeneous Feller process

For $x_{0}>S>0$, by virtue of (16) and (17), one has:

$$
g_{\lambda}\left(S \mid x_{0}\right)=\frac{f_{\lambda}\left(S \mid x_{0}\right)}{f_{\lambda}(S \mid S)}=\left(\frac{S}{x_{0}}\right)^{\beta / r-1} \exp \left\{\frac{\alpha\left(S-x_{0}\right)}{r}\right\} \frac{f_{\lambda}\left(x_{0} \mid S\right)}{f_{\lambda}(S \mid S)} .
$$

Then, making use of (10) in (18), for $x_{0}>S>0$, one obtains:

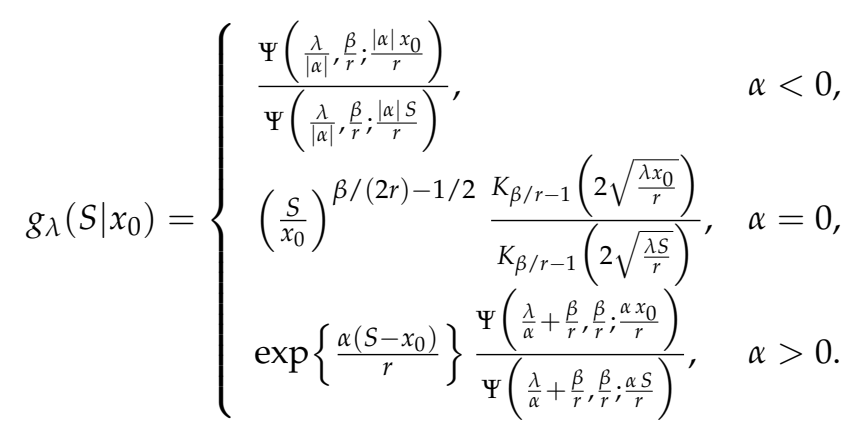


From (19), one derives the ultimate FPT probability through $S$ starting from $x_{0}$, with $x_{0}>S>0$ :

$P\left(S \mid x_{0}\right)=\int_{0}^{+\infty} g\left(S, t \mid x_{0}\right) d t= \begin{cases}1, & {[\alpha<0, \beta>0] \text { or }[\alpha=0,0<\beta \leq r],} \\ \left(\frac{S}{x_{0}}\right)^{\beta / r-1}, & \alpha=0, \beta>r, \\ \frac{\Gamma\left(1-\frac{\beta}{r}, \frac{\alpha x_{0}}{r}\right)}{\Gamma\left(1-\frac{\beta}{r}, \frac{\alpha S}{r}\right)}, & \alpha>0, \beta>0,\end{cases}$

with the use of (11) and (14). Furthermore, if $x_{0}>0$, taking the limit as $S \downarrow 0$ in (19), for $0<\beta<r$, one has:

$$
g_{\lambda}\left(0 \mid x_{0}\right)= \begin{cases}\frac{\Gamma\left(1-\frac{\beta}{r}+\frac{\lambda}{|\alpha|}\right)}{\Gamma\left(1-\frac{\beta}{r}\right)} \Psi\left(\frac{\lambda}{|\alpha|}, \frac{\beta}{r} ; \frac{|\alpha| x_{0}}{r}\right), & \alpha<0, \\ \frac{2}{\Gamma\left(1-\frac{\beta}{r}\right)}\left(\frac{\lambda x_{0}}{r}\right)^{1 / 2-\beta /(2 r)} K_{\beta / r-1}\left(2 \sqrt{\frac{\lambda x_{0}}{r}}\right), & \alpha=0, \\ \exp \left\{-\frac{\alpha x_{0}}{r}\right\} \frac{\Gamma\left(1+\frac{\lambda}{\alpha}\right)}{\Gamma\left(1-\frac{\beta}{r}\right)} \Psi\left(\frac{\lambda}{\alpha}+\frac{\beta}{r}, \frac{\beta}{r} ; \frac{\alpha x_{0}}{r}\right), & \alpha>0,\end{cases}
$$

where the relation

$$
\Psi(a, c ; 0)=\frac{\Gamma(1-c)}{\Gamma(a-c+1)}, \quad 0<\operatorname{Re} c<1,
$$

has been used for $\alpha \neq 0$, whereas the identity

$$
\Gamma(a) \Gamma(1-a)=\frac{\pi}{\sin (a \pi)} \quad 0<a<1 .
$$

has been applied for $\alpha=0$. From (21), one obtains the ultimate FPT probability through zero state starting from $x_{0}$, with $x_{0}>0$ :

$$
P\left(0 \mid x_{0}\right)=\int_{0}^{+\infty} g\left(0, t \mid x_{0}\right) d t= \begin{cases}1, & \alpha \leq 0,0<\beta<r \\ 1-\frac{\gamma\left(1-\frac{\beta}{r}, \frac{\alpha x_{0}}{r}\right)}{\Gamma\left(1-\frac{\beta}{r}\right)}, & \alpha>0,0<\beta<r\end{cases}
$$

where

$$
\gamma(a, x)=\int_{0}^{x} t^{a-1} e^{-t} d t=\Gamma(a)-\Gamma(a, x), \quad \operatorname{Re} a>0,
$$

denotes the incomplete gamma function.

For $x_{0}>0$ and $0<\beta<r$, the inverse LT of $g_{\lambda}\left(0 \mid x_{0}\right)$, given in (21), can be explicitly evaluated:

$$
g\left(0, t \mid x_{0}, t_{0}\right)= \begin{cases}\frac{1}{\left(t-t_{0}\right) \Gamma\left(1-\frac{\beta}{r}\right)}\left(\frac{x_{0}}{r\left(t-t_{0}\right)}\right)^{1-\beta / r} \exp \left\{-\frac{x_{0}}{r\left(t-t_{0}\right)}\right\}, & \alpha=0, \\ \frac{1}{\Gamma\left(1-\frac{\beta}{r}\right)} \frac{\alpha}{e^{\alpha\left(t-t_{0}\right)}-1}\left[\frac{\alpha x_{0} e^{\alpha\left(t-t_{0}\right)}}{r\left(e^{\alpha\left(t-t_{0}\right)}-1\right)}\right]^{1-\beta / r} \exp \left\{-\frac{\alpha x_{0} e^{\alpha\left(t-t_{0}\right)}}{r\left(e^{\left(\left(t-t_{0}\right)\right.}-1\right)}\right\}, & \alpha \neq 0 .\end{cases}
$$

Indeed, since (cf. Erdelyi et al. [49], p. 283, no. 35)

$$
\lambda^{-v / 2} K_{v}(2 \sqrt{a \lambda})=\frac{a^{-v / 2}}{2} \int_{0}^{+\infty} e^{-\lambda t} t^{\nu-1} e^{-a / t} d t, \quad a>0,
$$

the start of (23) follows from (21) for $\alpha=0$. Moreover, for $\alpha \neq 0$ making use of the first of (14) in (21) and recalling that (cf. Tricomi [48], p. 90)

$$
\Psi(s, c ; z)=\frac{1}{\Gamma(s)} \int_{0}^{+\infty} e^{-s \tau} \exp \left\{-\frac{z}{e^{\tau}-1}\right\}\left(1-e^{-\tau}\right)^{-c} d \tau, \quad \operatorname{Re} z>0, \operatorname{Re} s>0,
$$


the second part of (23) is obtained.

In Figure 1, the FPT pdf $g\left(0, t \mid x_{0}, t_{0}\right)$, given in (23), is plotted as function of $t$ for some choices of $\alpha$ and $r$, with $\beta=r / 2$.

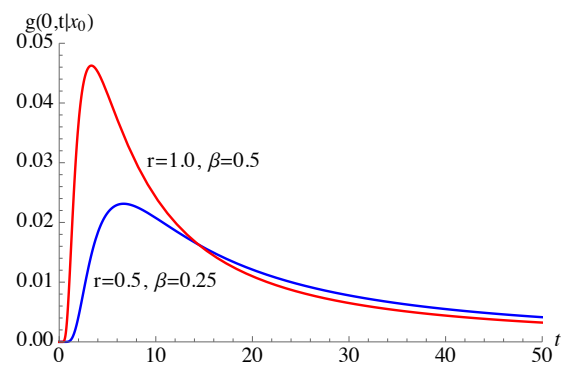

(a)

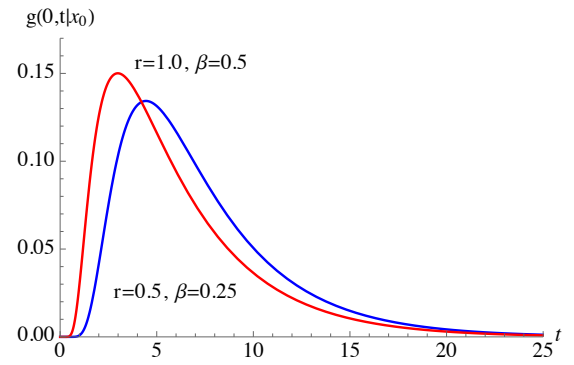

(b)

Figure 1. The FPT pdf (23) is plotted as function of $t$ for $t_{0}=0, x_{0}=5$. (a) FPT pdf for $\alpha=0$. (b) FPT pdf for $\alpha=-0.5$.

(b) FPT upwards for the time-homogeneous Feller process

By virtue of (10), from (17), for $0<x_{0}<S$, one has

$$
g_{\lambda}\left(S \mid x_{0}\right)=\frac{f_{\lambda}\left(S \mid x_{0}\right)}{f_{\lambda}(S \mid S)}= \begin{cases}\frac{\Phi\left(\frac{\lambda}{|\alpha|}, \frac{\beta}{r} ; \frac{\alpha \alpha \mid x_{0}}{r}\right)}{\Phi\left(\frac{\lambda}{|\alpha|}, \frac{\beta}{r} ; \frac{\alpha \alpha \mid S}{r}\right)}, & \alpha<0, \\ \left(\frac{S}{x_{0}}\right)^{\beta /(2 r)-1 / 2} \frac{I_{\beta / r-1}\left(2 \sqrt{\frac{\lambda x_{0}}{r}}\right)}{I_{\beta / r-1}\left(2 \sqrt{\frac{\lambda S}{r}}\right)}, & \alpha=0, \\ \exp \left\{\frac{\alpha\left(S-x_{0}\right)}{r}\right\} \frac{\Phi\left(\frac{\lambda}{\alpha}+\frac{\beta}{r}, \frac{\beta}{r} ; \frac{\alpha x_{0}}{r}\right)}{\Phi\left(\frac{\lambda}{\alpha}+\frac{\beta}{r}, \frac{\beta}{r} ; \frac{\alpha S}{r}\right)}, & \alpha>0,\end{cases}
$$

whereas for $x_{0}=0$ and $S>0$, it results that:

$$
g_{\lambda}(S \mid 0)=\frac{f_{\lambda}(S \mid 0)}{f_{\lambda}(S \mid S)}= \begin{cases}\frac{1}{\Phi\left(\frac{\lambda}{|\alpha|} ; \frac{\beta}{r} ; \frac{|\alpha| S}{r}\right)}, & \alpha<0, \\ \frac{1}{\Gamma\left(\frac{\beta}{r}\right)}\left(\frac{\lambda S}{r}\right)^{\beta /(2 r)-1 / 2} \frac{1}{I_{\beta / r-1}\left(2 \sqrt{\frac{\lambda S}{r}}\right)}, & \alpha=0, \\ \exp \left\{\frac{\alpha S}{r}\right\} \frac{1}{\Phi\left(\frac{\lambda}{\alpha}+\frac{\beta}{r}, \frac{\beta}{r} ; \frac{\alpha S}{r}\right)}, & \alpha>0 .\end{cases}
$$

From (24) and (25), one derives that the first passage through $S$ starting from $x_{0}$ is a sure event, i.e.,

$$
P\left(S \mid x_{0}\right)=\int_{0}^{+\infty} g\left(S, t \mid x_{0}\right) d t=1, \quad 0 \leq x_{0}<S .
$$

\subsection{Relations between the FPT Densities for the Feller and the Wiener Processes}

The FPT pdf $g\left(S, t \mid x_{0}, t_{0}\right)$ for the time-homogeneous Feller process can be explicitly obtained for $\alpha=0$ and $\beta=r / 2$ or for $\alpha=0$ and $\beta=3 r / 2$, as proved in Proposition 1 and in Proposition 2, respectively. Moreover, in these cases, there is a relationship between the FPT pdf of Feller process and the FPT pdf of the standard Wiener process.

Proposition 1. Let $X(t)$ be a time-homogeneous Feller diffusion process, having $B_{1}(x)=r / 2$ and $B_{2}(x)=2 r x$, with a zero-flux condition in the zero state. 
- If $x_{0}>S \geq 0$, one has:

$$
g\left(S, t \mid x_{0}, t_{0}\right)=\frac{\sqrt{x_{0}}-\sqrt{S}}{\sqrt{\pi r\left(t-t_{0}\right)^{3}}} \exp \left\{-\frac{\left(\sqrt{x_{0}}-\sqrt{S}\right)^{2}}{r\left(t-t_{0}\right)}\right\}
$$

and $P\left(S \mid x_{0}\right)=1$.

- If $0 \leq x_{0}<S$, one obtains:

$$
\begin{aligned}
& g\left(S, t \mid x_{0}, t_{0}\right)=\frac{\sqrt{S}-\sqrt{x_{0}}}{\sqrt{\pi r\left(t-t_{0}\right)^{3}}} \exp \left\{-\frac{\left(\sqrt{S}-\sqrt{x_{0}}\right)^{2}}{r\left(t-t_{0}\right)}\right\} \\
& \times\left\{1+2 \sum_{j=1}^{+\infty}(-1)^{j} \exp \left\{-\frac{4 j^{2} S}{r\left(t-t_{0}\right)}\right\}\left[\cosh \left(\frac{4 j \sqrt{S}\left(\sqrt{S}-\sqrt{x_{0}}\right)}{r\left(t-t_{0}\right)}\right)\right.\right. \\
& \left.\left.-\frac{2 j \sqrt{S}}{\sqrt{S}-\sqrt{x_{0}}} \sinh \left(\frac{4 j \sqrt{S}\left(\sqrt{S}-\sqrt{x_{0}}\right)}{r\left(t-t_{0}\right)}\right)\right]\right\},
\end{aligned}
$$

or alternatively

$$
\begin{aligned}
g\left(S, t \mid x_{0}, t_{0}\right)= & \frac{\pi r}{4 S} \sum_{n=1}^{+\infty}(-1)^{n-1}(2 n-1) \exp \left\{-\frac{(2 n-1)^{2} \pi^{2} r\left(t-t_{0}\right)}{16 S}\right\} \\
& \times \cos \left[\frac{(2 n-1) \pi}{2} \sqrt{\frac{x_{0}}{S}}\right]
\end{aligned}
$$

and $P\left(S \mid x_{0}\right)=1$

Proof. We assume that $\alpha=0$ and $\beta=r / 2$. In this case, the zero state is a regular reflecting boundary. Making use of the relations (cf. Abramowitz and Stegun [50], p. 443, no. 10.2.14 and p. 444, no. 10.2.17)

$$
I_{-1 / 2}(x)=\sqrt{\frac{2}{\pi}} \frac{\cosh (x)}{\sqrt{x}}, \quad K_{-1 / 2}(x)=\sqrt{\frac{\pi}{2 x}} e^{-x},
$$

from (19), (21), (24) and (25) with $\alpha=0$ and $\beta=r / 2$, it follows that:

$$
g_{\lambda}\left(S \mid x_{0}\right)= \begin{cases}\exp \left\{-\sqrt{2 \lambda}\left(\sqrt{\frac{2 x_{0}}{r}}-\sqrt{\frac{2 S}{r}}\right)\right\}, & x_{0}>S \geq 0, \\ \frac{\cosh \left(2 \sqrt{\frac{\lambda x_{0}}{r}}\right)}{\cosh \left(2 \sqrt{\frac{\lambda S}{r}}\right)}, & 0 \leq x_{0}<S .\end{cases}
$$

When $x_{0}>S \geq 0$, the right-hand side of (30) identifies with the LT $g_{\lambda}^{(W)}\left(\sqrt{2 S / r} \mid \sqrt{2 x_{0} / r}\right)$ of the FPT pdf $g_{W}$ through $\sqrt{2 S / r}$ for a standard Wiener process originated in $\sqrt{2 x_{0} / r}$. Hence, for $\alpha=0$ and $\beta=r / 2$, one has

$$
g\left(S, t \mid x_{0}, t_{0}\right)=g_{W}\left(\sqrt{\frac{2 S}{r}}, t \mid \sqrt{\frac{2 x_{0}}{r}}, t_{0}\right), \quad x_{0}>S \geq 0,
$$

from which (27) follows. Instead, for $0 \leq x_{0}<S$, the right-hand side of (30) is the LT $\gamma_{\lambda}^{(W)}\left(\sqrt{2 S / r} \mid \sqrt{2 x_{0} / r}\right)$ of the FPT pdf $\gamma_{W}$ through $\sqrt{2 S / r}$ for a standard Wiener process, starting from $\sqrt{2 x_{0} / r}$, restricted to $[0,+\infty)$ with 0 reflecting boundary (cf., for instance, Giorno and Nobile [3]). Then, for $\alpha=0$ and $\beta=r / 2$, one obtains:

$$
g\left(S, t \mid x_{0}, t_{0}\right)=\gamma_{W}\left(\sqrt{\frac{2 S}{r}}, t \mid \sqrt{\frac{2 x_{0}}{r}}, t_{0}\right), \quad 0 \leq x_{0}<S,
$$


from which (28) follows. The alternative expression (29) is derived by performing the inverse LT to the second expression in (30) and by using formula 33.149, p. 190 in Spiegel et al.'s work [51].

We note that by setting $S=0$ in (27) we obtain (23) with $\alpha=0$ and $\beta=r / 2$.

In Figure 2, the FPT pdf (28) is plotted as function of $t$ for $t_{0}=0, x_{0}=5$ and various choices of parameters $r$ and $S$.

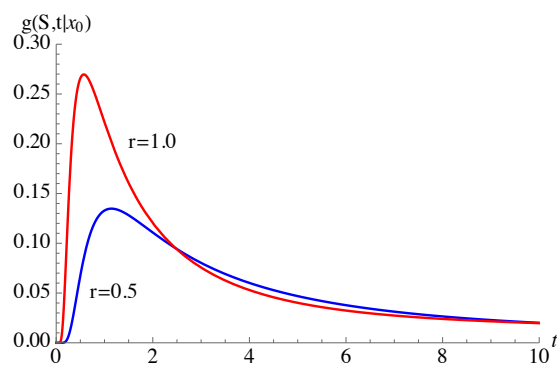

(a)

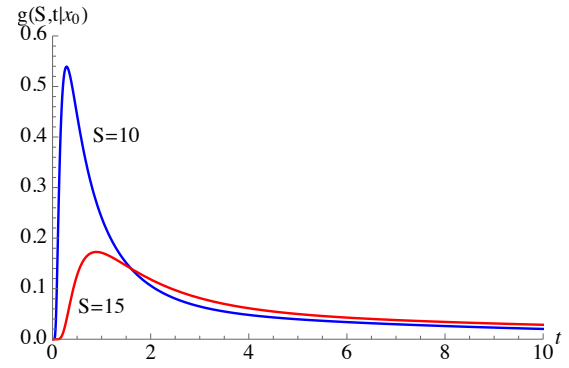

(b)

Figure 2. The FPT pdf (28) is plotted as function of $t$ for $t_{0}=0$ and $x_{0}=5$. (a) FPT pdf for $S=10$. (b) FPT pdf for $r=2$.

Proposition 2. Let $X(t)$ be a time-homogeneous Feller diffusion process, having $B_{1}(x)=3 r / 2$ and $B_{2}(x)=2 r x$, with a zero-flux condition in the zero state.

- If $x_{0}>S>0$, one has:

$$
g\left(S, t \mid x_{0}, t_{0}\right)=\sqrt{\frac{S}{x_{0}}} \frac{\sqrt{x_{0}}-\sqrt{S}}{\sqrt{\pi r\left(t-t_{0}\right)^{3}}} \exp \left\{-\frac{\left(\sqrt{x_{0}}-\sqrt{S}\right)^{2}}{r\left(t-t_{0}\right)}\right\}
$$

and $P\left(S \mid x_{0}\right)=\sqrt{S / x_{0}}$.

- If $0<x_{0}<S$, one obtains:

$$
\begin{gathered}
g\left(S, t \mid x_{0}, t_{0}\right)=\sqrt{\frac{S}{x_{0}}} \frac{\sqrt{S}-\sqrt{x_{0}}}{\sqrt{\pi r\left(t-t_{0}\right)^{3}}} \exp \left\{-\frac{\left(\sqrt{S}-\sqrt{x_{0}}\right)^{2}}{r\left(t-t_{0}\right)}\right\} \\
\quad \times\left\{1+2 \sum_{j=1}^{+\infty} \exp \left\{-\frac{4 j^{2} S}{r\left(t-t_{0}\right)}\right\}\left[\cosh \left(\frac{4 j \sqrt{S}\left(\sqrt{S}-\sqrt{x_{0}}\right)}{r\left(t-t_{0}\right)}\right)\right.\right. \\
\left.\left.-\frac{2 j \sqrt{S}}{\sqrt{S}-\sqrt{x_{0}}} \sinh \left(\frac{4 j \sqrt{S}\left(\sqrt{S}-\sqrt{x_{0}}\right)}{r\left(t-t_{0}\right)}\right)\right]\right\}
\end{gathered}
$$

or alternatively

$$
g\left(S, t \mid x_{0}, t_{0}\right)=\frac{\pi r}{2 \sqrt{x_{0} S}} \sum_{n=1}^{+\infty}(-1)^{n+1} n \exp \left\{-\frac{n^{2} \pi^{2} r\left(t-t_{0}\right)}{4 S}\right\} \sin \left(n \pi \sqrt{\frac{x_{0}}{S}}\right)
$$

and $P\left(S \mid x_{0}\right)=1$.

- If $x_{0}=0$ and $S>0$, one has:

$$
\begin{gathered}
g\left(S, t \mid 0, t_{0}\right)=\frac{4 \sqrt{S}}{\sqrt{\pi r\left(t-t_{0}\right)^{3}}} \exp \left\{-\frac{S}{r\left(t-t_{0}\right)}\right\} \sum_{j=1}^{+\infty} j \exp \left\{-\frac{4 j^{2} S}{r\left(t-t_{0}\right)}\right\} \\
\times\left[\frac{4 j S}{r\left(t-t_{0}\right)} \cosh \left(\frac{4 j S}{r\left(t-t_{0}\right)}\right)-\left(1+\frac{2 S}{r\left(t-t_{0}\right)}\right) \sinh \left(\frac{4 j S}{r\left(t-t_{0}\right)}\right)\right],
\end{gathered}
$$


or alternatively

$$
g\left(S, t \mid 0, t_{0}\right)=\frac{\pi^{2} r}{2 S} \sum_{n=1}^{+\infty}(-1)^{n+1} n^{2} \exp \left\{-\frac{n^{2} \pi^{2} r\left(t-t_{0}\right)}{4 S}\right\}
$$

and $P\left(S \mid x_{0}\right)=1$.

Proof. We assume that $\alpha=0$ and $\beta=3 r / 2$. In this case, the zero state is an entrance boundary. Making use of the relations (cf. Abramowitz and Stegun [50], p. 443, no. 10.2.13 and p. 444, no. 10.2.17)

$$
I_{1 / 2}(x)=\sqrt{\frac{2}{\pi}} \frac{\sinh (x)}{\sqrt{x}}, \quad K_{1 / 2}(x)=\sqrt{\frac{\pi}{2 x}} e^{-x},
$$

from (19), (24) and (25) with $\alpha=0$ and $\beta=3 r / 2$, it follows that:

$$
g_{\lambda}\left(S \mid x_{0}\right)= \begin{cases}\sqrt{\frac{S}{x_{0}}} \exp \left\{-\sqrt{2 \lambda}\left(\sqrt{\frac{2 x_{0}}{r}}-\sqrt{\frac{2 S}{r}}\right)\right\}, & x_{0}>S>0, \\ \sqrt{\frac{S}{x_{0}}} \frac{\sinh \left(2 \sqrt{\frac{\lambda x_{0}}{r}}\right)}{\sinh \left(2 \sqrt{\frac{\lambda S}{r}}\right)}, & 0<x_{0}<S, \\ 2 \sqrt{\frac{\lambda S}{r}} \frac{1}{\sinh \left(2 \sqrt{\frac{\lambda S}{r}}\right)}, & x_{0}=0, S>0 .\end{cases}
$$

We note that when $x_{0}>S>0$, the right-hand side of (36) identifies with the LT $\sqrt{S / x_{0}} g_{\lambda}^{(W)}\left(\sqrt{2 S / r} \mid \sqrt{2 x_{0} / r}\right)$ of the function $\sqrt{S / x_{0}} g_{W}$, where $g_{W}$ is the FPT pdf through $\sqrt{2 S / r}$ of a standard Wiener process originated in $\sqrt{2 x_{0} / r}$. Hence, for $\alpha=0$ and $\beta=3 r / 2$, one has

$$
g\left(S, t \mid x_{0}, t_{0}\right)=\sqrt{\frac{S}{x_{0}}} g_{W}\left(\sqrt{\frac{2 S}{r}}, t \mid \sqrt{\frac{2 x_{0}}{r}}, t_{0}\right), \quad x_{0}>S>0,
$$

that leads to (32). Instead, for $0<x_{0}<S$ the right-hand side of (36) is the LT $\sqrt{S / x_{0}} h_{\lambda}^{(W)}\left(\sqrt{2 S / r} \mid \sqrt{2 x_{0} / r}\right)$ of the function $\sqrt{S / x_{0}} h_{W}$, where $h_{W}$ is the first-exit time pdf through $\sqrt{2 S / r}$ for a standard Wiener process, starting from $\sqrt{2 x_{0} / r}$, defined in $(0,+\infty)$ with 0 absorbing boundary (cf., for instance, Giorno and Nobile [3]). Then, for $\alpha=0$ and $\beta=3 r / 2$, one has

$$
g\left(S, t \mid x_{0}, t_{0}\right)=\sqrt{\frac{S}{x_{0}}} h_{W}\left(\sqrt{\frac{2 S}{r}}, t \mid \sqrt{\frac{2 x_{0}}{r}}, t_{0}\right), \quad 0<x_{0}<S,
$$

from which (32) follows. The alternative expression (33) can be obtained by performing the inverse LT to the second expression in (36) and by using formula 33.148, p. 190 in Spiegel et al. [51] (by changing the sign). Finally, (34) and (35) follow by taking the limit as $x_{0} \downarrow 0$ in (32) and (33), respectively.

In Figure 3, the FPT pdf (32) is plotted as function of $t$ for $t_{0}=0, x_{0}=5$ and various choices of parameters $r$ and $S$. We note that, due to the different nature of the zero state, the peaks of FPT densities of Figure 3 are more pronounced with respect to those of Figure 2. 


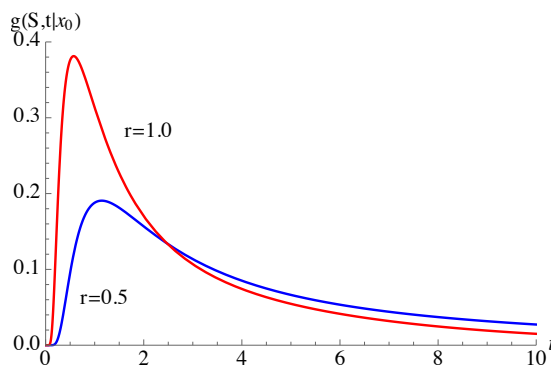

(a)

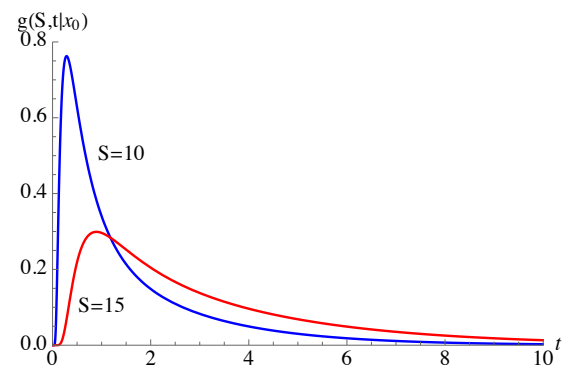

(b)

Figure 3. The FPT pdf (32) is plotted as function of $t$ for $t_{0}=0, x_{0}=5$. (a) FPT pdf for $S=10$. (b) FPT pdf for $r=2$.

\subsection{Relations between the FPT Densities for the Feller and the Ornstein-Uhlenbeck Processes}

For $\alpha \neq 0$ and $\beta=r / 2$ or $\alpha \neq 0$ and $\beta=3 r / 2$, the FPT pdf $g\left(S, t \mid x_{0}, t_{0}\right)$ of the Feller process can be related to the FPT pdf of the Ornstein-Uhlenbeck process.

Proposition 3. Let $X(t)$ be a time-homogeneous Feller diffusion process, having $B_{1}(x)=\alpha x+r / 2$ and $B_{2}(x)=2 r x(\alpha \neq 0)$, with a zero-flux condition in the zero state.

- If $x_{0}>S \geq 0$, one has:

$$
g_{\lambda}\left(S \mid x_{0}\right)= \begin{cases}\exp \left\{\frac{|\alpha|\left(x_{0}-S\right)}{2 r}\right\} \frac{D_{-2 \lambda /|\alpha|}\left(\sqrt{\frac{2|\alpha| x_{0}}{r}}\right)}{D_{-2 \lambda /|\alpha|}\left(\sqrt{\frac{2|\alpha| S}{r}}\right)}, \quad \alpha<0, \\ \exp \left\{-\frac{\alpha\left(x_{0}-S\right)}{2 r}\right\} \frac{D_{-1-2 \lambda / \alpha}\left(\sqrt{\frac{2 \alpha x_{0}}{r}}\right)}{D_{-1-2 \lambda / \alpha}\left(\sqrt{\frac{2 \alpha S}{r}}\right)}, \quad \alpha>0,\end{cases}
$$

where $D_{v}(x)$ denotes the parabolic cylinder function, and

$$
P\left(S \mid x_{0}\right)= \begin{cases}1, & \alpha<0 \\ \frac{1-\operatorname{Erf}\left(\sqrt{\frac{\alpha x_{0}}{r}}\right)}{1-\operatorname{Erf}\left(\sqrt{\frac{\alpha S}{r}}\right)}, & \alpha>0\end{cases}
$$

where $\operatorname{Erf}(x)=(2 / \sqrt{\pi}) \int_{0}^{+\infty} e^{-z^{2}} d z$ denotes the error function.

- If $0 \leq x_{0}<S$, one obtains:

$$
g_{\lambda}\left(S \mid x_{0}\right)= \begin{cases}\frac{\Phi\left(\frac{\lambda}{|\alpha|}, \frac{1}{2} ; \frac{|\alpha| x_{0}}{r}\right)}{\Phi\left(\frac{\lambda}{\mid \alpha}, \frac{1}{2} ; \frac{|\alpha| S}{r}\right)}, & \alpha<0, \\ \exp \left\{\frac{\alpha\left(S-x_{0}\right)}{r}\right\} \frac{\Phi\left(\frac{\lambda}{\alpha}+\frac{1}{2}, \frac{1}{2} ; \frac{\alpha x_{0}}{r}\right)}{\Phi\left(\frac{\lambda}{\alpha}+\frac{1}{2}, \frac{1}{2} ; \frac{\alpha S}{r}\right)}, & \alpha>0\end{cases}
$$

and $P\left(S \mid x_{0}\right)=1$.

Proof. Let $\alpha \neq 0$ and $\beta=r / 2$. We assume that the state $x=0$ is a regular reflecting boundary. Recalling that (cf. Tricomi [48], p. 219, no. (1)):

$$
D_{v}(x)=2^{v / 2} e^{-x^{2} / 4} \Psi\left(-\frac{v}{2}, \frac{1}{2} ; \frac{x^{2}}{2}\right), \quad \operatorname{Re} x>0,
$$


for $x_{0}>S>0$ from (19) one obtains (37). Furthermore, for $x_{0}>0$ and $S=0$, from (21) with $\alpha \neq 0$ and $\beta=r / 2$, making use of (40), we have

$$
g_{\lambda}\left(0 \mid x_{0}\right)= \begin{cases}\frac{2^{\lambda /|\alpha|}}{\sqrt{\pi}} \Gamma\left(\frac{1}{2}+\frac{\lambda}{|\alpha|}\right) \exp \left\{\frac{|\alpha| x_{0}}{2 r}\right\} D_{-2 \lambda /|\alpha|}\left(\sqrt{\frac{2|\alpha| x_{0}}{r}}\right), & \alpha<0, \\ \frac{2^{\lambda / \alpha+1 / 2}}{\sqrt{\pi}} \Gamma\left(1+\frac{\lambda}{\alpha}\right) \exp \left\{-\frac{\alpha x_{0}}{2 r}\right\} D_{-1-2 \lambda / \alpha}\left(\sqrt{\frac{2 \alpha x_{0}}{r}}\right), & \alpha>0 .\end{cases}
$$

Equation (41) identifies with (37) for $S=0$, being (cf. Tricomi [48], p. 221, no. (9)):

$$
D_{v}(0)=\frac{\sqrt{\pi} 2^{v / 2}}{\Gamma\left(\frac{1-v}{2}\right)} .
$$

Since (cf. Tricomi [48], p. 234, no. 15 and p. 235, no. 18):

$$
D_{0}(x)=\exp \left\{-\frac{x^{2}}{4}\right\}, \quad D_{-1}(x)=\sqrt{\frac{\pi}{2}} \exp \left\{\frac{x^{2}}{4}\right\}\left[1-\operatorname{Erf}\left(\frac{x}{\sqrt{2}}\right)\right],
$$

by setting $\lambda=0$ in (37), one obtains (38).

Instead, for $0 \leq x_{0}<S$, from (24) and (25), with $\alpha \neq 0$ and $\beta=r / 2$, one immediately obtains (39). Consequently, by setting $\lambda=0$ and making use of the second expression in (13), it follows that $P\left(S \mid x_{0}\right)=1$.

We note that, for $x_{0}>S \geq 0$, the right-hand side of (37) identifies with the LT $g_{\lambda}^{(\mathrm{OU})}\left(\sqrt{2 S / r} \mid \sqrt{2 x_{0} / r}\right)$ of the FPT pdf $g_{\mathrm{OU}}$ from $\sqrt{2 x_{0} / r}$ through $\sqrt{2 S / r}$ for the OrnsteinUhlenbeck process with infinitesimal drift $C_{1}(x)=\alpha x / 2$ and infinitesimal variance $C_{2}=1$. Hence, for $\alpha \neq 0$ and $\beta=r / 2$ from (37) one has:

$$
g\left(S, t \mid x_{0}, t_{0}\right)=g_{\mathrm{OU}}\left(\sqrt{\frac{2 S}{r}}, t \mid \sqrt{\frac{2 x_{0}}{r}}, t_{0}\right), \quad x_{0}>S \geq 0 .
$$

Furthermore, for $0 \leq x_{0}<S$ the right-hand side of (39) is the LT $\gamma_{\lambda}^{(\mathrm{OU})}\left(\sqrt{2 S / r} \mid \sqrt{2 x_{0} / r}\right)$ of the FPT pdf $\gamma_{\mathrm{OU}}$ from $\sqrt{2 x_{0} / r}$ to $\sqrt{2 S / r}$ for the Ornstein-Uhlenbeck process with infinitesimal drift $C_{1}(x)=\alpha x / 2$ and infinitesimal variance $C_{2}=1$, defined in $[0,+\infty)$, with 0 reflecting boundary. Therefore, for $\alpha \neq 0$ and $\beta=r / 2$ from (39), one obtains:

$$
g\left(S, t \mid x_{0}, t_{0}\right)=\gamma_{\mathrm{OU}}\left(\sqrt{\frac{2 S}{r}}, t \mid \sqrt{\frac{2 x_{0}}{r}}, t_{0}\right), \quad 0 \leq x_{0}<S .
$$

For $\alpha \neq 0$ and $\beta=r / 2$, relations (44) and (45) show that the FPT density of the Feller process can be also interpreted as the the FPT density of an Ornstein-Uhlenbeck process, that is known only when $S=0$. Therefore, from (44), one has:

$$
\begin{aligned}
& g\left(0, t \mid x_{0}, t_{0}\right)=g_{\mathrm{OU}}\left(0, t \mid \sqrt{\frac{2 x_{0}}{r}}, t_{0}\right) \\
& =\sqrt{\frac{x_{0} e^{\alpha\left(t-t_{0}\right)}}{r \pi}}\left[\frac{\alpha}{e^{\alpha\left(t-t_{0}\right)}-1}\right]^{3 / 2} \exp \left\{-\frac{\alpha x_{0} e^{\alpha\left(t-t_{0}\right)}}{r\left(e^{\alpha\left(t-t_{0}\right)}-1\right)}\right\}, \quad x_{0}>0,
\end{aligned}
$$

which identifies with (23) for $\alpha \neq 0$ and $\beta=r / 2$.

Proposition 4. Let $X(t)$ be a time-homogeneous Feller diffusion process, having $B_{1}(x)=\alpha x+3 r / 2$ and $B_{2}(x)=2 r x(\alpha \neq 0)$, with a zero-flux condition in the zero state. 
- If $x_{0}>S>0$, one has:

$$
g_{\lambda}\left(S \mid x_{0}\right)= \begin{cases}\sqrt{\frac{S}{x_{0}}} \exp \left\{\frac{|\alpha|\left(x_{0}-S\right)}{2 r}\right\} \frac{D_{1-2 \lambda /|\alpha|}\left(\sqrt{\frac{2|\alpha| x_{0}}{r}}\right)}{D_{1-2 \lambda /|\alpha|}\left(\sqrt{\frac{2|\alpha| S}{r}}\right)}, \quad \alpha<0, \\ \sqrt{\frac{S}{x_{0}}} \exp \left\{-\frac{\alpha\left(x_{0}-S\right)}{2 r}\right\} \frac{D_{-2-2 \lambda / \alpha}\left(\sqrt{\frac{2 \alpha x_{0}}{r}}\right)}{D_{-2-2 \lambda / \alpha}\left(\sqrt{\frac{2 \alpha S}{r}}\right)}, \quad \alpha>0\end{cases}
$$

and

$$
P\left(S \mid x_{0}\right)= \begin{cases}1, & \alpha<0, \\ \sqrt{\frac{S}{x_{0}}} \frac{1-\sqrt{\frac{\alpha x_{0} \pi}{r}} \exp \left\{\frac{\alpha x_{0}}{r}\right\}\left[1-\operatorname{Erf}\left(\sqrt{\frac{\alpha x_{0}}{r}}\right)\right]}{1-\sqrt{\frac{\alpha S \pi}{r}} \exp \left\{\frac{\alpha S}{r}\right\}\left[1-\operatorname{Erf}\left(\sqrt{\frac{\alpha S}{r}}\right)\right]}, \quad \alpha>0 .\end{cases}
$$

- If $0 \leq x_{0}<S$, one obtains:

$$
g_{\lambda}\left(S \mid x_{0}\right)= \begin{cases}\frac{\Phi\left(\frac{\lambda}{|\alpha|}, \frac{3}{2} ; \frac{|\alpha| x_{0}}{r}\right)}{\Phi\left(\frac{\lambda}{|\alpha|}, \frac{3}{2} ; \frac{|\alpha| S}{r}\right)} & \alpha<0, \\ \exp \left\{\frac{\alpha\left(S-x_{0}\right)}{r}\right\} \frac{\Phi\left(\frac{\lambda}{\alpha}+\frac{3}{2}, \frac{3}{2} ; \frac{\alpha x_{0}}{r}\right)}{\Phi\left(\frac{\lambda}{\alpha}+\frac{3}{2}, \frac{3}{2} ; \frac{\alpha S}{r}\right)}, & \alpha>0\end{cases}
$$

and $P\left(S \mid x_{0}\right)=1$.

Proof. Let $\alpha \neq 0$ and $\beta=3 r / 2$, so that the state $x=0$ is an entrance boundary. For $x_{0}>S>0$, recalling that (cf. Tricomi [48], p. 219, no. (2))

$$
D_{v}(x)=2^{(v-1) / 2} e^{-x^{2} / 4} x \Psi\left(\frac{1-v}{2}, \frac{3}{2} ; \frac{x^{2}}{2}\right), \quad \operatorname{Re} x>0,
$$

from (19), with $\alpha \neq 0$ and $\beta=3 r / 2$, one obtains (46). Moreover, making use of relation $D_{v+1}(x)=x D_{v}(x)-v D_{v-1}(x)$ and of (43), one has

$$
D_{1}(x)=x \exp \left\{-\frac{x^{2}}{4}\right\}, \quad D_{-2}(x)=\exp \left\{-\frac{x^{2}}{4}\right\}-\sqrt{\frac{\pi}{2}} x \exp \left\{\frac{x^{2}}{4}\right\}\left[1-\operatorname{Erf}\left(\frac{x}{\sqrt{2}}\right)\right],
$$

so that, by setting $\lambda=0$ in (46), one obtains (47).

Instead, for $0 \leq x_{0}<S$ from (24) and (25), with $\alpha \neq 0$ and $\beta=3 r / 2$, Equation (48) immediately follows. Finally, by setting $\lambda=0$ in (48) and making use of the second expression in (13), one has $P\left(S \mid x_{0}\right)=1$.

For $x_{0}>S>0$, we note that the right-hand side of (46) identifies with the LT $\sqrt{S / x_{0}} g_{\lambda+\alpha / 2}^{(\mathrm{OU})}\left(\sqrt{2 S / r} \mid \sqrt{2 x_{0} / r}\right)$ of $\sqrt{S / x_{0}} e^{-\alpha t / 2} g_{\mathrm{OU}}$, where $g_{\mathrm{OU}}$ is the FPT pdf from $\sqrt{2 x_{0} / r}$ through $\sqrt{2 S / r}$ for the Ornstein-Uhlenbeck process with infinitesimal drift $C_{1}(x)=\alpha x / 2$ and infinitesimal variance $C_{2}=1$. Hence, for $\alpha \neq 0$ and $\beta=3 r / 2$ one has:

$$
g\left(S, t \mid x_{0}, t_{0}\right)=\sqrt{\frac{S}{x_{0}}} \exp \left\{-\frac{\alpha\left(t-t_{0}\right)}{2}\right\} g_{\mathrm{OU}}\left(\sqrt{\frac{2 S}{r}}, t \mid \sqrt{\frac{2 x_{0}}{r}}, t_{0}\right), \quad x_{0}>S>0 .
$$

For $\alpha \neq 0$ and $\beta=3 r / 2$, Equation (51) shows that a functional relationship between the FPT densities of the Feller and Ornstein-Uhlenbeck processes exists. 


\section{FPT Moments for the Time-Homogeneous Feller Process}

When $P\left(S \mid x_{0}\right)=1$, the FPT moments of the time-homogeneous Feller process $X(t)$ with a zero-flux condition in the zero state

$$
t_{n}\left(S \mid x_{0}\right)=\int_{0}^{+\infty} t^{n} g\left(S, t \mid x_{0}\right) d t, \quad n=1,2, \ldots
$$

can be evaluated via $g_{\lambda}\left(S \mid x_{0}\right)$ as:

$$
t_{n}\left(S \mid x_{0}\right)=\left.(-1)^{n} \frac{d^{n} g_{\lambda}\left(S \mid x_{0}\right)}{d \lambda^{n}}\right|_{\lambda=0^{\prime}} \quad n=1,2, \ldots
$$

We note that the computation of higher order derivatives becomes more and more laborious, making this procedure impractical for the Feller process. An alternative method is based on Siegert's iterative formulas (cf. Siegert [52]) that hold for time-homogeneous diffusion processes. In particular, when $P\left(S \mid x_{0}\right) \equiv t_{0}\left(S \mid x_{0}\right)=1$, Siegert's iterative formulas for the Feller process are the following:

- If $S<x_{0}$, then

$$
t_{n}\left(S \mid x_{0}\right)=n \int_{S}^{x_{0}} d z h(z) \int_{z}^{+\infty} s(u) t_{n-1}(S \mid u) d u, \quad n=1,2, \ldots
$$

- If $S>x_{0}$, then

$$
t_{n}\left(S \mid x_{0}\right)=n \int_{x_{0}}^{S} d z h(z) \int_{0}^{z} s(u) t_{n-1}(S \mid u) d u, \quad n=1,2, \ldots
$$

with $h(x)$ and $s(x)$ defined in (4).

\subsection{Mean of FPT Downwards}

We distinguish the cases $x_{0}>S>0$ and $x_{0}>0, S=0$.

If $x_{0}>S>0$ and $[\alpha<0, \beta>0]$ or $[\alpha=0,0<\beta \leq r]$, we have proved in (20) that $P\left(S \mid x_{0}\right)=1$, so that from (52) for $\alpha=0$ and $0<\beta \leq r$ one has that $t_{1}\left(S \mid x_{0}\right)$ diverges, whereas if $\alpha<0$ and $\beta>0$ one obtains:

$$
\begin{aligned}
t_{1}\left(S \mid x_{0}\right)=\frac{1}{|\alpha|} \Gamma\left(\frac{\beta}{r}\right) \int_{|\alpha| S / r}^{|\alpha| x_{0} / r} z^{-\beta / r} e^{z} d z-\frac{1}{\beta} \sum_{n=0}^{+\infty} \frac{1}{(1+\beta / r)_{n}}\left(\frac{|\alpha|}{r}\right)^{n} \frac{x_{0}^{n+1}-S^{n+1}}{n+1} \\
x_{0}>S>0 .
\end{aligned}
$$

Moreover, for $x_{0}>0$ and $S=0$, due to (22), $P\left(0 \mid x_{0}\right)=1$ if and only if $\alpha \leq 0$ and $0<\beta<r$. Making use of (22), for $\alpha=0$ and $0<\beta<r$, one has that $t_{1}\left(0 \mid x_{0}\right)$ diverges, whereas for $\alpha<0$ and $0<\beta<r$ the FPT mean is

$$
\begin{aligned}
t_{1}\left(0 \mid x_{0}\right)= & \frac{1}{|\alpha|} \Gamma\left(\frac{\beta}{r}\right) \sum_{n=0}^{+\infty} \frac{1}{n !} \frac{1}{n+1-\beta / r}\left(\frac{|\alpha| x_{0}}{r}\right)^{n+1-\beta / r} \\
& -\frac{1}{\beta} \sum_{n=0}^{+\infty} \frac{1}{(1+\beta / r)_{n}}\left(\frac{|\alpha|}{r}\right)^{n} \frac{x_{0}^{n+1}}{n+1}, \quad x_{0}>0 .
\end{aligned}
$$

In Figure 4, the FPT mean (54) is plotted for $x_{0}=5, S=3$ and $\alpha=-0.5$ for different choices of $\beta$ and $r$. We note that $t_{1}\left(S \mid x_{0}\right)$ decreases as $r$ increases, whereas it increases with $\beta$. 


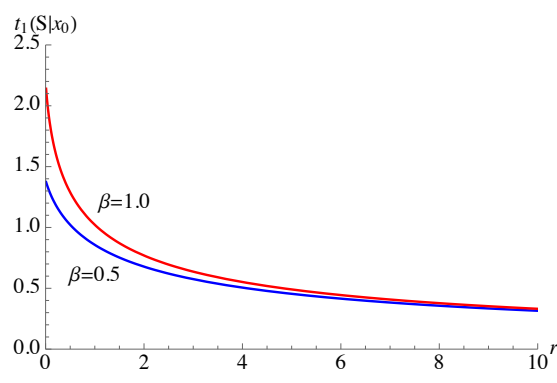

(a)

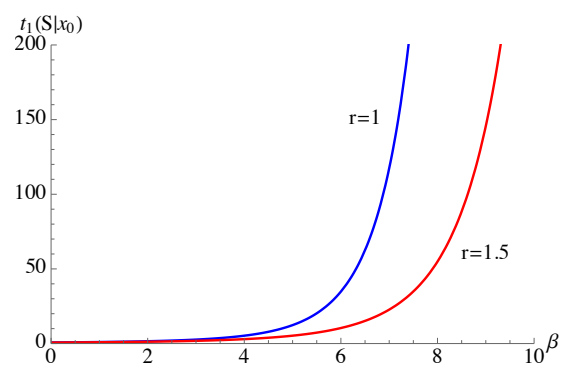

(b)

Figure 4. The FPT mean (54) is plotted for $x_{0}=5, S=3$ and $\alpha=-0.5$. (a) FPT mean as function of $r$. (b) FPT mean as function of $\beta$.

\subsection{Moments of FPT Upwards}

If $0 \leq x_{0}<S$, we have proved in (26) that $P\left(S \mid x_{0}\right)=1$, so that from (53) one has:

$$
\begin{aligned}
t_{1}\left(S \mid x_{0}\right)= & \frac{1}{\beta} \sum_{n=0}^{+\infty} \frac{1}{(1+\beta / r)_{n}}\left(-\frac{\alpha}{r}\right)^{n} \frac{S^{n+1}-x_{0}^{n+1}}{n+1}, \\
t_{2}\left(S \mid x_{0}\right)= & \frac{2}{\beta} t_{1}\left(S \mid x_{0}\right) \sum_{n=0}^{+\infty} \frac{1}{(1+\beta / r)_{n}}\left(-\frac{\alpha}{r}\right)^{n} \frac{S^{n+1}}{n+1} \\
& -\frac{2 r}{\beta} \sum_{n=1}^{+\infty} \frac{1}{(1+\beta / r)_{n}}\left(-\frac{\alpha}{r}\right)^{n+1} \frac{S^{n+1}-x_{0}^{n+1}}{n+1} \sum_{i=1}^{n} \frac{1}{i}, \\
t_{3}\left(S \mid x_{0}\right)= & \frac{3}{\beta} t_{2}\left(S \mid x_{0}\right) \sum_{n=0}^{+\infty} \frac{1}{(1+\beta / r)_{n}}\left(-\frac{\alpha}{r}\right)^{n} \frac{S^{n+1}}{n+1} \\
& -\frac{6 r}{\beta} t_{1}\left(S \mid x_{0}\right) \sum_{n=1}^{+\infty} \frac{1}{(1+\beta / r)_{n}}\left(-\frac{\alpha}{r}\right)^{n+1} \frac{S^{n+1}}{n+1} \sum_{i=1}^{n} \frac{1}{i} \\
& -\frac{6 r}{\beta} \sum_{n=2}^{+\infty} \frac{1}{(1+\beta / r)_{n+2}}\left(-\frac{\alpha}{r}\right)^{n+1} \frac{S^{n+1}-x_{0}^{n+1}}{n+1} \sum_{i=2}^{n} \frac{1}{i} \sum_{j=2}^{i} \frac{1}{j-1} .
\end{aligned}
$$

In particular, when $\alpha<0$, from (56) it follows that:

- $t_{1}\left(S \mid x_{0}\right)$ decreases as $r$ increases and $\lim _{r \rightarrow+\infty} t_{1}\left(S \mid x_{0}\right)=\left(S-x_{0}\right) / \beta$;

- $\quad t_{1}\left(S \mid x_{0}\right)$ decreases as $\beta$ increases and $\lim _{\beta \rightarrow+\infty} t_{1}\left(S \mid x_{0}\right)=0$.

In Figure 5, the FPT mean (54) is plotted for $x_{0}=5, S=10$ and $\alpha=-0.5$ for several choices of $\beta$ and $r$.

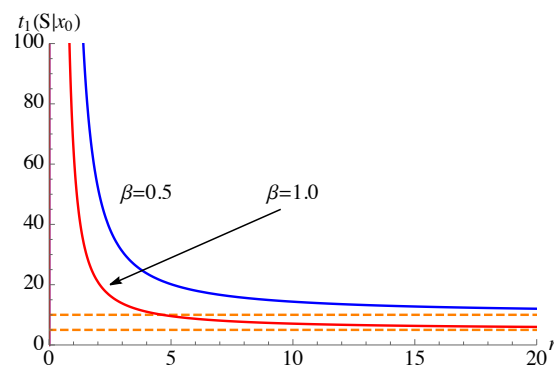

(a)

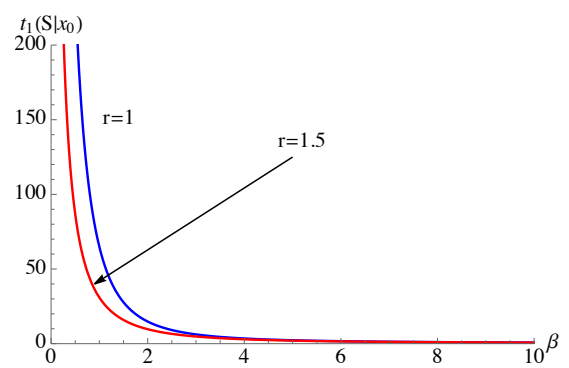

(b)

Figure 5. The FPT mean (54) is plotted for $x_{0}=5, S=10$ and $\alpha=-0.5$. (a) FPT mean as function of $r$, the dashed lines indicate the asymptotic limit $\left(S-x_{0}\right) / \beta$. (b) FPT mean as function of $\beta$.

The expressions (56) of the FPT moments are very complicated and do not allow us to highlight the quantitative behavior of the moments as a function of the involved 
parameters. Nevertheless, some unexpected features can be discovered as a result of systematic computations in which the mean $t_{1}\left(S \mid x_{0}\right)$, the variance $\operatorname{Var}\left(S \mid x_{0}\right)=t_{2}\left(S \mid x_{0}\right)-$ $t_{1}^{2}\left(S \mid x_{0}\right)$, the coefficient of variation $\mathrm{Cv}\left(S \mid x_{0}\right)=\sqrt{\operatorname{Var}\left(S \mid x_{0}\right)} / t_{1}\left(S \mid x_{0}\right)$ and the skewness

$$
\Sigma\left(S \mid x_{0}\right)=\frac{t_{3}\left(S \mid x_{0}\right)-3 t_{1}\left(S \mid x_{0}\right) t_{2}\left(S \mid x_{0}\right)+2 t_{1}^{3}\left(S \mid x_{0}\right)}{\left[\operatorname{Var}\left(S \mid x_{0}\right)\right]^{3 / 2}}
$$

of the FPT are evaluated. In Table $1, t_{1}\left(S \mid x_{0}\right) \operatorname{Var}\left(S \mid x_{0}\right), \mathrm{Cv}\left(S \mid x_{0}\right)$ and $\Sigma\left(S \mid x_{0}\right)$ are listed for various boundaries and initial states, with $\alpha=-0.5, \beta=0.2$ and $r=1$. As shown in Table 1, for large boundaries the coefficient of variation of FPT approaches the value 1 and the skewness of the FPT approaches the value 2. Hence, when $\alpha<0$, it is argued that the FPT pdf of the Feller process is susceptible to an exponential approximation for a wide range of constant boundaries $S$ and of initial states $x_{0}$, with $S>x_{0}$. This property does not occur when $\alpha \geq 0$. Table 1 also shows that the values of $t_{1}\left(S \mid x_{0}\right)$ and $\operatorname{Var}\left(S \mid x_{0}\right)$ become insensitive to the starting point $x_{0}$ of the process as the boundary $S$ increases.

Table 1. For the Feller process, with $B_{1}(x)=-0.5 x+0.2$ and $B_{2}(x)=2 x$, the mean, the variance, the coefficient of variation and the skewness of FPT are listed for $x_{0}=0,5,10$ and for increasing values of the boundary $S>x_{0}$.

\begin{tabular}{cccccc}
\hline & $S$ & $\boldsymbol{t}_{\mathbf{1}}(\boldsymbol{S} \mid \boldsymbol{x} \mathbf{0})$ & $\boldsymbol{V a r}\left(\boldsymbol{S} \mid \boldsymbol{x}_{\mathbf{0}}\right)$ & $\mathbf{C v}(\boldsymbol{S} \mid \boldsymbol{x} \mathbf{0})$ & $\boldsymbol{\Sigma}\left(\boldsymbol{S} \mid \boldsymbol{x}_{\mathbf{0}}\right)$ \\
\hline$x_{0}=0$ & 5 & $9.111607 \times 10^{1}$ & $7.918037 \times 10^{3}$ & 0.976593 & 1.999485 \\
& 10 & $1.030455 \times 10^{3}$ & $1.054126 \times 10^{6}$ & 0996362 & 1.999973 \\
& 20 & $1.305581 \times 10^{5}$ & $1.704399 \times 10^{10}$ & 0.999958 & 1.998950 \\
& 30 & $1.771815 \times 10^{7}$ & $3.139316 \times 10^{14}$ & 0.999998 & 1.999116 \\
& 40 & $2.472975 \times 10^{9}$ & $6.115604 \times 10^{18}$ & 1.000000 & 1.998936 \\
& 50 & $3.502187 \times 10^{11}$ & $1.226522 \times 10^{23}$ & 0.999996 & 2.037217 \\
$x_{0}=5$ & 60 & $5.004295 \times 10^{13}$ & $2.504289 \times 10^{27}$ & 0.999998 & 2.002647 \\
& 70 & $7.194172 \times 10^{15}$ & $5.175608 \times 10^{31}$ & 1.000000 & 1.999006 \\
& 10 & $9.393392 \times 10^{2}$ & $1.046208 \times 10^{6}$ & 1.088896 & 2.021401 \\
& 20 & $1.304670 \times 10^{5}$ & $1.704398 \times 10^{10}$ & 1.000656 & 1.999551 \\
& 30 & $1.771805 \times 10^{7}$ & $3.139325 \times 10^{14}$ & 1.000005 & 1.998926 \\
& 40 & $2.472975 \times 10^{9}$ & $6.115603 \times 10^{18}$ & 1.000000 & 1.997063 \\
$x_{0}=10$ & 50 & $3.502187 \times 10^{11}$ & $1.226531 \times 10^{23}$ & 1.000000 & 2.036733 \\
& 60 & $5.004295 \times 10^{13}$ & $2.504283 \times 10^{27}$ & 0.999997 & 2.002072 \\
& 70 & $7.194173 \times 10^{15}$ & $5.175608 \times 10^{31}$ & 1.000000 & 2.000006 \\
& 15 & $1.041999 \times 10^{4}$ & $1.299491 \times 10^{8}$ & 1.094005 & 2.022940 \\
& 20 & $1.295276 \times 10^{5}$ & $1.704293 \times 10^{10}$ & 1.007882 & 1.999778 \\
& 30 & $1.771711 \times 10^{7}$ & $3.139304 \times 10^{14}$ & 1.000055 & 1.998418 \\
& 40 & $2.472974 \times 10^{9}$ & $6.115604 \times 10^{18}$ & 1.000000 & 1.999088 \\
& 50 & $3.502187 \times 10^{11}$ & $1.226532 \times 10^{23}$ & 1.000000 & 1.981817 \\
& 60 & $5.004295 \times 10^{13}$ & $2.504274 \times 10^{27}$ & 0.999995 & 2.002118 \\
& 70 & $7.194173 \times 10^{15}$ & $5.175605 \times 10^{31}$ & 0.999999 & 2.000089 \\
\hline
\end{tabular}

\section{Asymptotic Behavior of the FPT Density for the Time-Homogeneous Feller Process}

In Sections 2 and 3, we analyzed the FPT problem for a time-homogeneous Feller process and we assumed that the boundary $S$ is constant. Nevertheless, the inclusion of a time-varying boundary $S(t)$ is often useful to model various aspects of the time varying behavior of dynamic systems.

Let $S(t) \in C^{1}\left[t_{0},+\infty\right)$, with $S(t)>0$, where $C^{1}\left[t_{0},+\infty\right)$ denotes the set of continuously differentiable functions on $\left[t_{0},+\infty\right)$. For a time-homogeneous diffusion process, having drift $B_{1}(x)$ and infinitesimal variance $B_{2}(x)$, the FPT pdf $g\left[S(t), t \mid x_{0}, t_{0}\right]$ is the solution of the second-kind non-singular Volterra integral equation (cf. Buonocore [8]):

$g\left[S(t), t \mid x_{0}, t_{0}\right]=\varrho\left\{-2 \Omega\left[S(t), t \mid x_{0}, t_{0}\right]+2 \int_{t_{0}}^{t} g\left[S(u), u \mid x_{0}, t_{0}\right] \Omega[S(t), t \mid S(u), u] d u\right\}$ 
with $\varrho=1$ if $x_{0}<S\left(t_{0}\right)$ and $\varrho=-1$ if $x_{0}>S\left(t_{0}\right)$, and where

$$
\begin{aligned}
\Omega[S(t), t \mid z, \vartheta]=\frac{1}{2}\{ & \left.S^{\prime}(t)-B_{1}[S(t)]+\frac{3}{4} B_{2}^{\prime}[S(t)]\right\} f[S(t), t \mid z, \vartheta] \\
& +\left.\frac{1}{2} B_{2}[S(t)] \frac{\partial}{\partial x} f(x, t \mid z, \vartheta)\right|_{x=S(t)} .
\end{aligned}
$$

The knowledge of the transition pdf $f\left(x, t \mid x_{0}, t_{0}\right)$ of the considered diffusion process allows the formulation of effective numerical procedures to obtain $g\left[S(t), t \mid x_{0}, t_{0}\right]$ via (57) (cf., for instance, Buonocore et al. [8], Di Nardo et al. [10]).

For the Feller process, having $B_{1}(x)=\alpha x+\beta$ and $B_{2}(x)=2 r x$, with a zero-flux condition in the zero state, recalling (5) and (6), for $S(t)>0$ from (58), one obtains:

$$
\begin{aligned}
& \Omega[S(t), t \mid z, \vartheta]=\frac{1}{r(t-\vartheta)}\left[\frac{S(t)}{z}\right]^{(\beta-r) /(2 r)} \exp \left\{-\frac{S(t)+z}{r(t-\vartheta)}\right\}\left\{\frac{1}{2}\left[S^{\prime}(t)-\frac{2 S(t)}{t-\vartheta}+\beta-\frac{r}{2}\right]\right. \\
& \left.\quad \times I_{\beta / r-1}\left[\frac{2 \sqrt{z S(t)}}{r(t-\vartheta)}\right]+\frac{\sqrt{z S(t)}}{t-\vartheta} I_{\beta / r}\left[\frac{2 \sqrt{z S(t)}}{r(t-\vartheta)}\right]\right\}, \quad \alpha=0, \\
& \left.\Omega[S(t), t \mid z, \vartheta]=\frac{\alpha}{r\left(e^{\alpha(t-\vartheta)}-1\right)}\left[\frac{S(t) e^{-\alpha(t-\vartheta)}}{z}\right]\right]^{(\beta-r) /(2 r)} \exp \left\{-\frac{\alpha\left[S(t)+z e^{\alpha(t-\vartheta)}\right]}{r\left(e^{\alpha(t-\vartheta)}-1\right)}\right\} \\
& \quad \times\left\{\frac{1}{2}\left[S^{\prime}(t)-\alpha S(t)-\frac{2 \alpha S(t)}{e^{\alpha(t-\vartheta)}-1}+\beta-\frac{r}{2}\right] I_{\beta / r-1}\left[\frac{2 \alpha \sqrt{S(t) z e^{\alpha(t-\vartheta)}}}{r\left(e^{\alpha(t-\vartheta)}-1\right)}\right]\right. \\
& \left.\quad+\frac{\alpha \sqrt{S(t) z e^{\alpha(t-\vartheta)}}}{e^{\alpha(t-\vartheta)}-1} I_{\beta / r}\left[\frac{2 \alpha \sqrt{S(t) z e^{\alpha(t-\vartheta)}}}{r\left(e^{\alpha(t-\vartheta)}-1\right)}\right]\right\}, \quad \alpha \neq 0,
\end{aligned}
$$

where the relation (cf. Gradshteyn and Ryzhik [47], p. 928 no. 8.486.4)

$$
x \frac{d}{d x} I_{v}(x)=v I_{v}(x)+x I_{v+1}(x)
$$

has been used.

Let $0 \leq x_{0}<S\left(t_{0}\right)$. We focus our analysis on the asymptotic behavior of the FPT pdf for the Feller diffusion process, with $\alpha<0, \beta>0$ and $r>0$, by considering separately two cases: $S(t)$ is an asymptotically constant boundary and $S(t)$ is an asymptotically periodic boundary.

\subsection{Asymptotically Constant Boundary}

We consider the FPT problem for the Feller process through the asymptotically constant boundary

$$
S(t)=S+\eta(t)
$$

with $S(t)>0$, where $\eta(t) \in C^{1}\left[t_{0},+\infty\right)$ is a bounded function that does not depend on $S$, such that

$$
\lim _{t \rightarrow+\infty} \eta(t)=0, \quad \lim _{t \rightarrow+\infty} \frac{d \eta(t)}{d t}=0 .
$$

Since $\alpha<0$, the function $\Omega\left[S(t), t \mid x_{0}, t_{0}\right]$ approaches a constant value as $t \rightarrow+\infty$. Making use of (60), for $\alpha<0$, one has:

$$
\begin{aligned}
\zeta(S)= & -2 \lim _{t \rightarrow+\infty} \Omega\left[S(t), t \mid x_{0}, t_{0}\right]=-\left[B_{1}(S)-\frac{B_{2}^{\prime}(S)}{2}\right] W(S) \\
& =\frac{|\alpha| S-\beta+r / 2}{S \Gamma(\beta / r)}\left(\frac{|\alpha| S}{r}\right)^{\beta / r} \exp \left\{-\frac{|\alpha| S}{r}\right\}
\end{aligned}
$$


where (9) has been used. From (57), for $S \rightarrow+\infty$ and for large times the FPT density exhibits an exponential behavior (cf. Nobile et al. [12]). Specifically, for $\alpha<0$ and $S\left(t_{0}\right)>x_{0}$, one has:

$$
g\left[S(t), t \mid x_{0}, t_{0}\right] \simeq \zeta(S) e^{-\zeta(S)\left(t-t_{0}\right)}, \quad S>\frac{\beta-r / 2}{|\alpha|} .
$$

The goodness of the exponential approximation increases as the boundary progressively moves away from the starting point.

We now assume that the boundary $S(t)$ is constant, i.e., $S(t)=S>x_{0}$. By virtue of (53) for $n=1$, with $h(x)$ and $s(x)$ defined in (4), and recalling (63), for $\alpha<0$ and $S>x_{0}$ one has

$$
\lim _{S \rightarrow+\infty}\left[t_{1}\left(S \mid x_{0}\right) \zeta(S)\right]=1,
$$

implying that for $\alpha<0$ the FPT mean can be approximated by $1 / \zeta(S)$ for large values of $S$. Furthermore, by virtue of (64), for $\alpha<0$ and $S>x_{0}$, one obtains:

$$
t_{n}\left(S \mid x_{0}\right) \simeq m_{n}(S)=\frac{n !}{[\zeta(S)]^{n}}, \quad S \rightarrow+\infty, \quad n=1,2, \ldots
$$

In Table 2, the FPT moments $t_{i}\left(S \mid x_{0}\right)$ and their exponential approximations $m_{i}(S)$, with $i=1,2,3$, are listed for increasing values of the boundary $S>x_{0}=5$, showing a good degree of precision in the approximations. We emphasize that the exponential approximation of the FPT density (64) provides the growth trend of the FPT moments (65) for large constant boundaries $S$. Moreover, the goodness of the approximation depends on the parameters of the process that determine the exact shape of the FPT pdf.

\begin{tabular}{|c|c|c|c|c|c|c|}
\hline$S$ & $t_{1}(S \mid x 0)$ & $m_{1}(S)$ & $t_{2}(S \mid x 0)$ & $m_{2}(S)$ & $t_{3}(S \mid r)$ & $m_{3}(S)$ \\
\hline 10 & $9.393392 \times 10^{2}$ & $9.317407 \times 10^{2}$ & $1.9285660 \times 10^{6}$ & $1.736281 \times 10^{6}$ & $5.940178 \times 10^{9}$ & $4.853292 \times 10^{9}$ \\
\hline 15 & $1.135933 \times 10^{4}$ & $1.066806 \times 10^{4}$ & $2.6002970 \times 10^{8}$ & $2.276151 \times 10^{8}$ & $8.928760 \times 10^{12}$ & $7.284636 \times 10^{12}$ \\
\hline 20 & $1.304670 \times 10^{5}$ & $1.238882 \times 10^{5}$ & $3.406561 \times 10^{10}$ & $3.069659 \times 10^{10}$ & $1.334106 \times 10^{16}$ & $1.140884 \times 10^{16}$ \\
\hline 25 & $1.513230 \times 10^{6}$ & $1.451849 \times 10^{6}$ & $4.579980 \times 10^{12}$ & $4.215733 \times 10^{12}$ & $2.079027 \times 10^{19}$ & $1.836183 \times 10^{19}$ \\
\hline 30 & $1.771805 \times 10^{7}$ & $1.712069 \times 10^{7}$ & $6.278619 \times 10^{14}$ & $5.862362 \times 10^{14}$ & $3.336766 \times 10^{22}$ & $3.011031 \times 10^{22}$ \\
\hline 35 & $2.088298 \times 10^{8}$ & $2.028086 \times 10^{8}$ & $8.721976 \times 10^{16}$ & $8.226266 \times 10^{16}$ & $5.461092 \times 10^{25}$ & $5.005072 \times 10^{25}$ \\
\hline 40 & $2.472975 \times 10^{9}$ & $2.410683 \times 10^{9}$ & $1.223121 \times 10^{19}$ & $1.162278 \times 10^{19}$ & $9.069796 \times 10^{28}$ & $8.405655 \times 10^{28}$ \\
\hline 45 & $2.938886 \times 10^{10}$ & $2.873158 \times 10^{10}$ & $1.727408 \times 10^{21}$ & $1.651007 \times 10^{21}$ & $1.522551 \times 10^{32}$ & $1.423082 \times 10^{32}$ \\
\hline 50 & $3.502187 \times 10^{11}$ & $3.431753 \times 10^{11}$ & $2.453063 \times 10^{23}$ & $2.355386 \times 10^{23}$ & $2.593104 \times 10^{35}$ & $2.424931 \times 10^{35}$ \\
\hline 55 & $4.182641 \times 10^{12}$ & $4.106219 \times 10^{12}$ & $3.498881 \times 10^{25}$ & $3.372207 \times 10^{25}$ & $4.395433 \times 10^{38}$ & $4.154106 \times 10^{38}$ \\
\hline 60 & $5.004295 \times 10^{13}$ & $4.920524 \times 10^{13}$ & $5.008580 \times 10^{27}$ & $4.842311 \times 10^{27}$ & $7.521900 \times 10^{41}$ & $7.148012 \times 10^{41}$ \\
\hline 65 & $5.996341 \times 10^{14}$ & $5.903724 \times 10^{14}$ & $7.191219 \times 10^{29}$ & $6.970790 \times 10^{29}$ & $1.293204 \times 10^{45}$ & $1.234609 \times 10^{45}$ \\
\hline 70 & $7.194173 \times 10^{15}$ & $7.091026 \times 10^{15}$ & $1.035122 \times 10^{32}$ & $1.005653 \times 10^{32}$ & $2.234055 \times 10^{48}$ & $2.139334 \times 10^{48}$ \\
\hline 75 & $8.640679 \times 10^{16}$ & $8.525086 \times 10^{16}$ & $1.493226 \times 10^{34}$ & $1.453542 \times 10^{34}$ & $3.867332 \times 10^{51}$ & $3.717471 \times 10^{51}$ \\
\hline 80 & $1.038782 \times 10^{18}$ & $1.025758 \times 10^{18}$ & $2.158137 \times 10^{36}$ & $2.104357 \times 10^{36}$ & $6.724827 \times 10^{54}$ & $6.475681 \times 10^{54}$ \\
\hline 85 & $1.249855 \times 10^{19}$ & $1.235109 \times 10^{19}$ & $3.124278 \times 10^{38}$ & $3.050990 \times 10^{38}$ & $1.171392 \times 10^{58}$ & $1.130492 \times 10^{58}$ \\
\hline 90 & $1.504914 \times 10^{20}$ & $1.488148 \times 10^{20}$ & $4.529530 \times 10^{40}$ & $4.429166 \times 10^{40}$ & $2.044966 \times 10^{61}$ & $1.977376 \times 10^{61}$ \\
\hline 95 & $1.813196 \times 10^{21}$ & $1.794062 \times 10^{21}$ & $6.575356 \times 10^{42}$ & $6.437315 \times 10^{42}$ & $3.576723 \times 10^{64}$ & $3.464682 \times 10^{64}$ \\
\hline 100 & $2.185898 \times 10^{22}$ & $2.163987 \times 10^{22}$ & $9.556292 \times 10^{44}$ & $9.365678 \times 10^{44}$ & $6.267299 \times 10^{67}$ & $6.080161 \times 10^{67}$ \\
\hline
\end{tabular}

Table 2. For the time-homogeneous Feller process, with $B_{1}(x)=-0.5 x+0.2$ and $B_{2}(x)=2 x$, the FPT moments $t_{i}\left(S \mid x_{0}\right)$ and their exponential approximations $m_{i}(S)$, with $i=1,2,3$, are listed for increasing values of the boundary $S>x_{0}=5$. 


\subsection{Asymptotically Periodic Boundary}

We consider the FPT problem for the Feller process through an asymptotically periodic boundary $S(t)=S+\eta(t)$, with $S(t)>0$, where $\eta(t) \in C^{1}\left[t_{0},+\infty\right)$ is a bounded function, that does not depend on $S$, such that

$$
\lim _{k \rightarrow+\infty} \eta(t+k Q)=V(t), \quad \lim _{k \rightarrow+\infty} \frac{d \eta(t+k Q)}{d t}=\frac{d V(t)}{d t}
$$

with $V(t)$ being a periodic function of period $Q>0$ satisfying the condition:

$$
\int_{0}^{Q} V(u) d u=0 .
$$

Since $\alpha<0$, the function $\Omega\left[S(t+k Q), t+k Q \mid x_{0}, t_{0}\right]$ approaches a periodic function as $k \rightarrow+\infty$. Indeed, making use of (60) and recalling (9), for $\alpha<0$, one obtains:

$$
\begin{aligned}
& \zeta(S, t)=-2 \lim _{k \rightarrow+\infty} \Omega\left[S(t+k Q), t+k Q \mid x_{0}, t_{0}\right] \\
& \quad=-\left\{V^{\prime}(t)+B_{1}[S+V(t)]-\frac{B_{2}^{\prime}[S+V(t)]}{4}\right\} W[S+V(t)] \\
& \quad=\frac{|\alpha|[S+V(t)]-V^{\prime}(t)-\beta+r / 2}{[S+V(t)] \Gamma(\beta / r)}\left(\frac{|\alpha|[S+V(t)]}{r}\right)^{\beta / r} \exp \left\{-\frac{|\alpha|[S+V(t)]}{r}\right\} .
\end{aligned}
$$

By virtue of (57), for $S \rightarrow+\infty$ and for large times, the FPT density shows a nonhomogeneous exponential behavior. Specifically, for $\alpha<0$ and $S\left(t_{0}\right)>x_{0}$, one has:

$$
g\left[S(t), t \mid x_{0}, t_{0}\right] \simeq \zeta(S, t) \exp \left\{-\int_{t_{0}}^{t} \zeta(S, \vartheta) d \vartheta\right\}, \quad S>\frac{V^{\prime}(t)+\beta-r / 2}{|\alpha|}-V(t) .
$$

Hence, for $\alpha<0$, the FPT pdf of the Feller process through an asymptotically periodic boundary exhibits damped oscillations taking the form of a sequence of periodically spaced peaks whose amplitudes exponentially decrease.

\section{First-Passage Time for a Time-Inhomogeneous Feller-Type Process}

We consider the time-inhomogeneous Feller-type diffusion process $X(t)$ with infinitesimal drift and infinitesimal variance

$$
B_{1}(x, t)=\alpha(t) x+\xi r(t), \quad B_{2}(x, t)=2 r(t) x,
$$

defined in the state space $[0,+\infty)$, with $\alpha(t) \in \mathbb{R}, r(t)>0$ and $\xi>0$, with a zero-flux condition in the zero state. In the sequel, we denote by

$$
A\left(t \mid t_{0}\right)=\int_{t_{0}}^{t} \alpha(z) d z, \quad R\left(t \mid t_{0}\right)=\int_{t_{0}}^{t} r(\tau) e^{-A\left(\tau \mid t_{0}\right)} d \tau .
$$

\subsection{Transition Density}

The transition pdf $f\left(x, t \mid x_{0}, t_{0}\right)$ of $X(t)$ is solution of the Fokker-Planck equation

$$
\frac{\partial f\left(x, t \mid x_{0}, t_{0}\right)}{\partial t}=-\frac{\partial}{\partial x}\left\{[\alpha(t) x+\xi r(t)] f\left(x, t \mid x_{0}, t_{0}\right)\right\}+r(t) \frac{\partial^{2}}{\partial x^{2}}\left[x f\left(x, t \mid x_{0}, t_{0}\right)\right],
$$

to solve imposing the initial delta condition

$$
\lim _{t \downarrow t_{0}} f\left(x, t \mid x_{0}, t_{0}\right)=\delta\left(x-x_{0}\right)
$$


and the zero-flux condition in the zero state:

$$
\lim _{x \downarrow 0}\left\{[\alpha(t) x+\xi r(t)] f\left(x, t \mid x_{0}, t_{0}\right)-r(t) \frac{\partial}{\partial x}\left[x f\left(x, t \mid x_{0}, t_{0}\right)\right]\right\}=0 .
$$

By virtue of the transformations (cf. Capocelli and Ricciardi [53])

$$
\begin{aligned}
& \widehat{x}=x e^{-A(t \mid 0)}, \quad \widehat{x}_{0}=x_{0} e^{-A\left(t_{0} \mid 0\right),} \\
& \widehat{t}=R(t \mid 0)=h(t), \quad \widehat{t}_{0}=R\left(t_{0} \mid 0\right)=h\left(t_{0}\right), \\
& f\left(x, t \mid x_{0}, t_{0}\right)=e^{-A(t \mid 0)} \widehat{f}\left(\widehat{x}, \widehat{t} \mid \widehat{x}_{0}, \widehat{t}_{0}\right),
\end{aligned}
$$

the Fokker-Plank equation (71) and the conditions (72) and (73) lead to the Fokker-Planck equation of a time-homogeneous Feller process $\{Y(t), t \geq 0\}$ with infinitesimal drift $C_{1}=\xi$ and infinitesimal variance $C_{2}(\widehat{x})=2 \widehat{x}$, with a delta initial condition and a zero-flux condition in the zero state:

$$
\begin{aligned}
& \frac{\partial \widehat{f}\left(\widehat{x}, \widehat{t} \mid \widehat{x}_{0}, \hat{t}_{0}\right)}{\partial \hat{t}}=-\xi \frac{\partial \widehat{f}\left(\widehat{x}, \hat{t} \mid \widehat{x}_{0}, \widehat{t}_{0}\right)}{\partial \widehat{x}}+\frac{\partial^{2}}{\partial \widehat{x}^{2}}\left[\widehat{x} \widehat{f}\left(\widehat{x}, \widehat{t} \mid \widehat{x}_{0}, \widehat{t}_{0}\right)\right], \\
& \lim _{\widehat{t} \downarrow \widehat{t}_{0}} \widehat{f}\left(\widehat{x}, \widehat{t} \mid \widehat{x}_{0}, \widehat{t}_{0}\right)=\delta\left(\widehat{x}-\widehat{x}_{0}\right), \\
& \lim _{\widehat{x} \downarrow 0}\left\{\xi \widehat{f}\left(\widehat{x}, \widehat{t} \mid \widehat{x}_{0}, \widehat{t}_{0}\right)-\frac{\partial}{\partial \widehat{x}}\left[\widehat{x} \widehat{f}\left(\widehat{x}, \widehat{t} \mid \widehat{x}_{0}, \widehat{t}_{0}\right)\right]\right\}=0 .
\end{aligned}
$$

Note that if $0<\xi<1$ the zero state for $Y(t)$ is a regular reflecting boundary, whereas for $\xi \geq 1$ the state zero is an entrance boundary. Recalling (5) with $\beta=\xi$ and $r=1$, from (74) we obtain the transition pdf of the Feller-type diffusion process (69) with a zero-flux condition in the zero state:

$$
f\left(x, t \mid x_{0}, t_{0}\right)= \begin{cases}\frac{1}{x \Gamma(\xi)}\left[\frac{x e^{-A\left(t \mid t_{0}\right)}}{R\left(t \mid t_{0}\right)}\right]^{\xi} \exp \left\{-\frac{x e^{-A\left(t \mid t_{0}\right)}}{R\left(t \mid t_{0}\right)}\right\}, & x_{0}=0, \\ \frac{e^{-A\left(t \mid t_{0}\right)}}{R\left(t \mid t_{0}\right)}\left[\frac{x e^{-A\left(t \mid t_{0}\right)}}{x_{0}}\right]^{(\xi-1) / 2} \exp \left\{-\frac{x_{0}+x e^{-A\left(t \mid t_{0}\right)}}{R\left(t \mid t_{0}\right)}\right\} & \\ \quad \times I_{\xi-1}\left[\frac{2 \sqrt{x x_{0} e^{-A\left(t \mid t_{0}\right)}}}{R\left(t \mid t_{0}\right)}\right], & x_{0}>0,\end{cases}
$$

where we have used the relation:

$$
h(t)-h\left(t_{0}\right)=e^{-A\left(t_{0} \mid 0\right)} R\left(t \mid t_{0}\right) .
$$

When

$$
\lim _{t \rightarrow+\infty} A\left(t \mid t_{0}\right)=-\infty, \quad \lim _{t \rightarrow+\infty} R\left(t \mid t_{0}\right)=+\infty, \quad \lim _{t \rightarrow+\infty} \frac{\alpha(t)}{r(t)}=-\gamma, \quad \gamma>0,
$$

the Feller-type diffusion process (69), with a zero-flux condition in the zero state, allows a steady-state density:

$$
W(x)=\lim _{t \rightarrow+\infty} f\left(x, t \mid x_{0}, t_{0}\right)=\frac{(\gamma x)^{\xi}}{x \Gamma(\xi)} e^{-\gamma x}, \quad x>0,
$$

which is a gamma density of parameters $\xi$ and $1 / \gamma$. The steady-state density $W(x)$ is a decreasing function of $x$ when $\xi \leq 1$, whereas $W(x)$ has a single maximum in $x=(\xi-1) / \gamma$ for $\xi>1$.

The asymptotic behavior of the transition pdf of $X(t)$ when $\alpha(t)$ or $r(t)$ or both are periodic functions is discussed in Giorno and Nobile [33]. 


\subsection{FPT Densities}

The FPT pdf $g\left[S(t), t \mid x_{0}, t_{0}\right]$ of $X(t)$, defined in (69), can be written in terms of the FPT $\operatorname{pdf} \widehat{g}\left[\widehat{S}(\hat{t}), \widehat{t} \mid \widehat{x}_{0}, \widehat{t}_{0}\right]$ of the process $Y(t)$, having infinitesimal drift $C_{1}=\xi$ and infinitesimal variance $C_{2}(\widehat{x})=2 \widehat{x}$, with a zero-flux condition in the zero state. Indeed, recalling (74), one has

$$
g\left[S(t), t \mid x_{0}, t_{0}\right]=\frac{d h(t)}{d t} \widehat{g}\left\{\widehat{S}[h(t)], h(t) \mid \widehat{x}_{0}, h\left(t_{0}\right)\right\},
$$

where $\widehat{S}[h(t)]=S(t) e^{-A(t \mid 0)}$.

Proposition 5. For the diffusion process (69), with $0<\xi<1$, one has:

$$
g\left(0, t \mid x_{0}, t_{0}\right)=\frac{1}{\Gamma(1-\xi)} \frac{r(t) e^{-A\left(t \mid t_{0}\right)}}{R\left(t \mid t_{0}\right)}\left[\frac{x_{0}}{R\left(t \mid t_{0}\right)}\right]^{1-\xi} \exp \left\{-\frac{x_{0}}{R\left(t \mid t_{0}\right)}\right\}, \quad x_{0}>0,
$$

with $R\left(t \mid t_{0}\right)$ given in (70). Furthermore, the ultimate FPT probability is:

$$
\int_{0}^{+\infty} g\left(0, t \mid x_{0}, t_{0}\right) d t= \begin{cases}1, & \lim _{t \rightarrow+\infty} R\left(t \mid t_{0}\right)=+\infty, \\ 1-\frac{\gamma\left(1-\xi, x_{0} / c\right)}{\Gamma(1-\xi)}, & \lim _{t \rightarrow+\infty} R\left(t \mid t_{0}\right)=c<+\infty,\end{cases}
$$

with $\gamma(a, x)$ denoting the incomplete gamma function.

Proof. Relation (80) follows from (23) with $\alpha=0, \beta=\xi$ and $r=1$, making use of (74) and (79) with $S(t)=0$. Furthermore, (81) can be obtained by integrating (80) with $t$ in $\left(t_{0},+\infty\right)$.

Note that a general expression of the FPT density for the time-inhomogeneous Fellertype process (1) through the zero state is given by Giorno and Nobile [43].

In the following two propositions, we show that if $\xi=1 / 2$ or $\xi=3 / 2$, it is possible to obtain closed-form expressions for the FPT densities through the time-varying barrier $S(t)=S e^{A(t \mid 0)}$, with $S>0$.

Proposition 6. Let $X(t)$ be a time-inhomogeneous Feller-type diffusion process, having $B_{1}(x, t)=\alpha(t) x+r(t) / 2$ and $B_{2}(x, t)=2 r(t) x$, with $\alpha(t) \in \mathbb{R}, r(t)>0$ and a zero-flux condition in the zero state. We assume that $S(t)=S e^{A(t \mid 0)}$, with $S \geq 0$.

- If $x_{0}>S\left(t_{0}\right) \geq 0$, one has:

$$
g\left[S(t), t \mid x_{0}, t_{0}\right]=\frac{r(t) e^{-A\left(t \mid t_{0}\right)}}{\sqrt{\pi\left[R\left(t \mid t_{0}\right)\right]^{3}}}\left[\sqrt{x_{0}}-\sqrt{S\left(t_{0}\right)}\right] \exp \left\{-\frac{\left[\sqrt{x_{0}}-\sqrt{S\left(t_{0}\right)}\right]^{2}}{R\left(t \mid t_{0}\right)}\right\}
$$

and the ultimate FPT probability $P\left\{\mathcal{T}\left(x_{0}, t_{0}\right)<+\infty\right\}=1$ when $\lim _{t \rightarrow+\infty} R\left(t \mid t_{0}\right)=+\infty$.

- If $0 \leq x_{0}<S\left(t_{0}\right)$, one obtains:

$$
\begin{gathered}
g\left[S(t), t \mid x_{0}, t_{0}\right]=\frac{r(t) e^{-A\left(t \mid t_{0}\right)}}{\sqrt{\pi\left[R\left(t \mid t_{0}\right)\right]^{3}}}\left[\sqrt{S\left(t_{0}\right)}-\sqrt{x_{0}}\right] \exp \left\{-\frac{\left[\sqrt{S\left(t_{0}\right)}-\sqrt{x_{0}}\right]^{2}}{R\left(t \mid t_{0}\right)}\right\} \\
\times\left\{1+2 \sum_{j=1}^{+\infty}(-1)^{j} \exp \left\{-\frac{4 j^{2} S\left(t_{0}\right)}{R\left(t \mid t_{0}\right)}\right\}\left[\cosh \left(\frac{4 j \sqrt{S\left(t_{0}\right)}\left(\sqrt{S\left(t_{0}\right)}-\sqrt{x_{0}}\right)}{R\left(t \mid t_{0}\right)}\right)\right.\right. \\
\left.\left.-\frac{2 j \sqrt{S\left(t_{0}\right)}}{\sqrt{S\left(t_{0}\right)}-\sqrt{x_{0}}} \sinh \left(\frac{4 j \sqrt{S\left(t_{0}\right)}\left(\sqrt{S\left(t_{0}\right)}-\sqrt{x_{0}}\right)}{R\left(t \mid t_{0}\right)}\right)\right]\right\}
\end{gathered}
$$

and the ultimate FPT probability $P\left\{\mathcal{T}\left(x_{0}, t_{0}\right)<+\infty\right\}=1$ when $\lim _{t \rightarrow+\infty} R\left(t \mid t_{0}\right)=+\infty$. 
Proof. Relation (82) follows from (27) making use of (74) and (79). Indeed, for $0 \leq S\left(t_{0}\right)<x_{0}$, one has:

$$
g\left[S e^{A(t \mid 0)}, t \mid x_{0}, t_{0}\right]=\frac{r(t) e^{-A(t \mid 0)}\left(\sqrt{\widehat{x}_{0}}-\sqrt{S}\right)}{\sqrt{\pi\left[h(t)-h\left(t_{0}\right)\right]^{3}}} \exp \left\{-\frac{\left(\sqrt{\widehat{x}_{0}}-\sqrt{S}\right)^{2}}{h(t)-h\left(t_{0}\right)}\right\},
$$

from which, due to (76), (82) follows. Similarly, Equation (83) follows from (28), making use of (74), (76) and (79).

We note that, by setting $S(t)=0$ in (82), we obtain (80) for $\xi=1 / 2$.

Example 1. We consider the Feller-type process having $B_{1}(x, t)=\alpha x+r(t) / 2$ and $B_{2}(x, t)=2 r(t) x$, with

$$
r(t)=v\left[1+c \sin \left(\frac{2 \pi t}{Q}\right)\right], \quad t \geq 0,
$$

where $v>0$ is the average of the periodic function $r(t)$ of period $Q$ and $c$ is the amplitude of the oscillations, with $0 \leq c<1$. From (70), for $t \geq t_{0}$, one has $A\left(t \mid t_{0}\right)=\alpha\left(t-t_{0}\right)$ and

$$
R\left(t \mid t_{0}\right)= \begin{cases}v\left(t-t_{0}\right)+\frac{c v Q_{1}}{2 \pi}\left[\cos \left(\frac{2 \pi t_{0}}{Q}\right)-\cos \left(\frac{2 \pi t}{Q}\right)\right], & \alpha=0, \\ \frac{v}{\alpha}\left(1-e^{-\alpha\left(t-t_{0}\right)}\right)+\frac{c v Q}{4 \pi^{2}+Q^{2} \alpha^{2}}\left\{2 \pi \cos \left(\frac{2 \pi t_{0}}{Q_{1}}\right)\right. & \\ \left.+\alpha Q \sin \left(\frac{2 \pi t_{0}}{Q_{1}}\right)-e^{-\alpha\left(t-t_{0}\right)}\left[2 \pi \cos \left(\frac{2 \pi t}{Q}\right)+\alpha Q_{1} \sin \left(\frac{2 \pi t}{Q}\right)\right]\right\}, & \alpha \neq 0 .\end{cases}
$$

For $\alpha=-0.05, c=0.4$ and $Q=2$, in Figure 6, the FPT $p d f$ (83) from $x_{0}=5$ through $S(t)=S e^{\alpha t}$ is plotted as function of $t$ for different choices of $S$ and $v$.

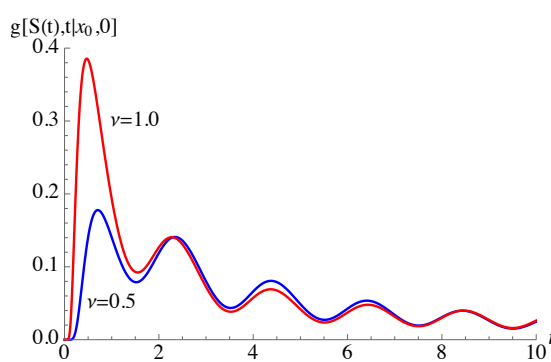

(a)

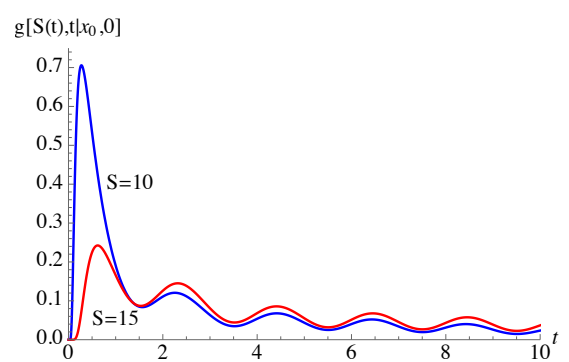

(b)

Figure 6. For the Feller-type process having $B_{1}(x, t)=-0.05 x+r(t) / 2$ and $B_{2}(x, t)=2 r(t) x$, with $r(t)=v[1+0.4 \sin (\pi t)]$, the FPT pdf (83) from $x_{0}=5$ through $S(t)=S e^{\alpha t}$ is plotted as a function of $t$. (a) FPT pdf for $S=10$. (b) FPT pdf for $v=2$.

Proposition 7. Let $X(t)$ be a time-inhomogeneous Feller-type diffusion process, having $B_{1}(x, t)=\alpha(t) x+3 r(t) / 2$ and $B_{2}(x, t)=2 r(t) x$, with $\alpha(t) \in \mathbb{R}, r(t)>0$ and a zero-flux condition in the zero state. We assume that $S(t)=S e^{A(t \mid 0)}$, with $S>0$.

- If $x_{0}>S\left(t_{0}\right)>0$, one has:

$$
\begin{gathered}
g\left[S(t), t \mid x_{0}, t_{0}\right]=\frac{r(t) e^{-A\left(t \mid t_{0}\right)}}{\sqrt{\pi\left[R\left(t \mid t_{0}\right)\right]^{3}}} \sqrt{\frac{S\left(t_{0}\right)}{x_{0}}}\left[\sqrt{x_{0}}-\sqrt{S\left(t_{0}\right)}\right] \exp \left\{-\frac{\left[\sqrt{x_{0}}-\sqrt{S\left(t_{0}\right)}\right]^{2}}{R\left(t \mid t_{0}\right)}\right\} \\
\quad \text { and } P\left\{\mathcal{T}\left(x_{0}, t_{0}\right)<+\infty\right\}=\sqrt{S\left(t_{0}\right) / x_{0}} \text { when } \lim _{t \rightarrow+\infty} R\left(t \mid t_{0}\right)=+\infty . \\
\quad \text { If } 0<x_{0}<S\left(t_{0}\right), \text { one obtains: }
\end{gathered}
$$




$$
\begin{gathered}
g\left[S(t), t \mid x_{0}, t_{0}\right]=\frac{r(t) e^{-A\left(t \mid t_{0}\right)}}{\sqrt{\pi\left[R\left(t \mid t_{0}\right)\right]^{3}}} \sqrt{\frac{S\left(t_{0}\right)}{x_{0}}}\left[\sqrt{S\left(t_{0}\right)}-\sqrt{x_{0}}\right] \exp \left\{-\frac{\left[\sqrt{S\left(t_{0}\right)}-\sqrt{x_{0}}\right]^{2}}{R\left(t \mid t_{0}\right)}\right\} \\
\times\left\{1+2 \sum_{j=1}^{+\infty} \exp \left\{-\frac{4 j^{2} S\left(t_{0}\right)}{R\left(t \mid t_{0}\right)}\right\}\left[\cosh \left(\frac{4 j \sqrt{S\left(t_{0}\right)}\left(\sqrt{S\left(t_{0}\right)}-\sqrt{x_{0}}\right)}{R\left(t \mid t_{0}\right)}\right)\right.\right. \\
\left.\left.-\frac{2 j \sqrt{S\left(t_{0}\right)}}{\sqrt{S\left(t_{0}\right)}-\sqrt{x_{0}}} \sinh \left(\frac{4 j \sqrt{S\left(t_{0}\right)}\left(\sqrt{S\left(t_{0}\right)}-\sqrt{x_{0}}\right)}{R\left(t \mid t_{0}\right)}\right)\right]\right\}
\end{gathered}
$$

and $P\left\{\mathcal{T}\left(x_{0}, t_{0}\right)<+\infty\right\}=1$ when $\lim _{t \rightarrow+\infty} R\left(t \mid t_{0}\right)=+\infty$.

- If $x_{0}=0$ and $S\left(t_{0}\right)>0$, one has:

$$
\begin{gathered}
g\left[S(t), t \mid 0, t_{0}\right]=\frac{4 r(t) e^{-A\left(t \mid t_{0}\right)} \sqrt{S\left(t_{0}\right)}}{\sqrt{\pi\left[R\left(t \mid t_{0}\right)\right]^{3}}} \exp \left\{-\frac{S\left(t_{0}\right)}{R\left(t \mid t_{0}\right)}\right\} \sum_{j=1}^{+\infty} j \exp \left\{-\frac{4 j^{2} S\left(t_{0}\right)}{R\left(t \mid t_{0}\right)}\right\} \\
\times\left[\frac{4 j S\left(t_{0}\right)}{R\left(t \mid t_{0}\right)} \cosh \left(\frac{4 j S\left(t_{0}\right)}{R\left(t \mid t_{0}\right)}\right)-\left(1+\frac{2 S\left(t_{0}\right)}{R\left(t \mid t_{0}\right)}\right) \sinh \left(\frac{4 j S\left(t_{0}\right)}{R\left(t \mid t_{0}\right)}\right)\right]
\end{gathered}
$$

and $P\left\{\mathcal{T}\left(x_{0}, t_{0}\right)<+\infty\right\}=1$ when $\lim _{t \rightarrow+\infty} R\left(t \mid t_{0}\right)=+\infty$.

Proof. Relations (87)-(89) follow from Proposition 2, making use of (74), (76) and (79).

Example 2. We consider the Feller-type process, having $B_{1}(x, t)=\alpha x+3 r(t) / 2$ and $B_{2}(x, t)=2 r(t) x$, with $r(t)$ given in (85). From (70), for $t \geq t_{0}$ one has $A\left(t \mid t_{0}\right)=\alpha\left(t-t_{0}\right)$ and $R\left(t \mid t_{0}\right)$ is given in (86). For $\alpha=-0.05, c=0.4$ and $Q=2$, in Figure 7, the FPT pdf (88) from $x_{0}=5$ through $S(t)=S e^{\alpha t}$ is plotted as function of $t$ for some choices of $S$ and $v$.

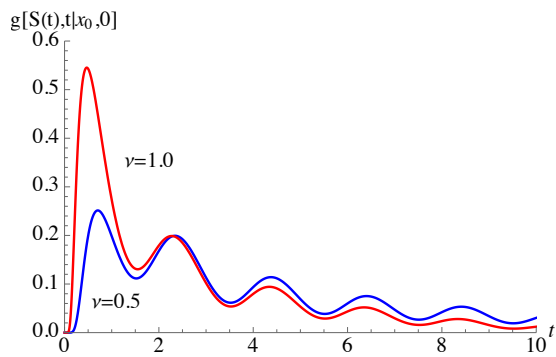

(a)

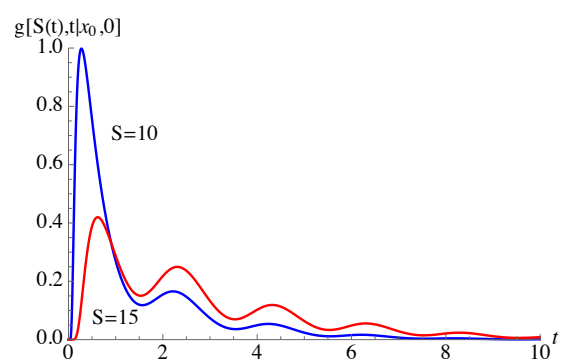

(b)

Figure 7. For the Feller-type process, having $B_{1}(x, t)=-0.05 x+3 r(t) / 2$ and $B_{2}(x, t)=2 r(t) x$, with $r(t)=v[1+0.4 \sin (\pi t)]$, the FPT pdf (88) from $x_{0}=5$ through $S(t)=S e^{\alpha t}$ is plotted as function of $t$. (a) FPT pdf for $S=10$. (b) FPT pdf for $v=2$.

\section{Asymptotic Behavior of the FPT Density for a Time-Inhomogeneous Feller-Type Process}

In the following proposition, we prove that the FPT density $g\left[S(t), t \mid x_{0}, t_{0}\right]$ of the process (69), with a zero-flux condition in the zero state, is a solution of a second-kind non-singular Volterra integral equation. 
Proposition 8. Let $S(t) \in C^{1}\left[t_{0},+\infty\right)$, with $S(t)>0$. For the time-inhomogeneous Feller-type diffusion process (69), with $\alpha(t) \in \mathbb{R}, r(t)>0$ and $\xi>0$, the FPT pdf $g\left[S(t), t \mid x_{0}, t_{0}\right]$ is a solution of the integral Equation (57) with $\varrho=1$ if $x_{0}<S\left(t_{0}\right)$ and $\varrho=-1$ if $x_{0}>S\left(t_{0}\right)$, where

$$
\begin{gathered}
\Omega[S(t), t \mid y, \tau]=\frac{r(t) e^{-A(t \mid \tau)}}{R(t \mid \tau)} \exp \left\{-\frac{S(t) e^{-A(t \mid \tau)}+y}{R(t \mid \tau)}\right\}\left[\frac{S(t) e^{-A(t \mid \tau)}}{y}\right]^{(\xi-1) / 2} \\
\times\left\{\frac{1}{2}\left[-\frac{\alpha(t) S(t)}{r(t)}+\frac{S^{\prime}(t)}{r(t)}-\frac{2 S(t) e^{-A(t \mid \tau)}}{R(t \mid \tau)}+\xi-\frac{1}{2}\right] I_{\xi-1}\left[\frac{2 \sqrt{y S(t) e^{-A(t \mid \tau)}}}{R(t \mid \tau)}\right]\right. \\
\left.+\frac{\sqrt{y S(t) e^{-A(t \mid \tau)}}}{R(t \mid \tau)} I_{\xi}\left[\frac{2 \sqrt{y S(t) e^{-A(t \mid \tau)}}}{R(t \mid \tau)}\right]\right\} .
\end{gathered}
$$

Proof. The FPT pdf $\widehat{g}\left[\widehat{S}(\widehat{t}), \widehat{t} \mid \widehat{x}_{0}, \widehat{t}_{0}\right]$ of the process $Y(t)$, with infinitesimal drift $C_{1}=\xi$ and infinitesimal variance $C_{2}(\widehat{x})=2 \widehat{x}$, with a zero-flux condition in the zero state, is a solution of the following integral equation

$$
\begin{aligned}
& \widehat{g}\left\{\widehat{S}[h(t)], h(t) \mid \widehat{x}_{0}, h\left(t_{0}\right)\right\}=\rho\left\{-2 \widehat{\Omega}\left\{\widehat{S}[h(t)], h(t) \mid \widehat{x}_{0}, h\left(t_{0}\right)\right\}\right. \\
& \left.\quad+2 \int_{h\left(t_{0}\right)}^{h(t)} \widehat{g}\left\{\widehat{S}(\vartheta), \vartheta \mid \widehat{x}_{0}, h\left(t_{0}\right)\right\} \widehat{\Omega}\{\widehat{S}[h(t)], h(t) \mid \widehat{S}(\vartheta), \vartheta\} d \vartheta\right\}, \quad \widehat{x}_{0} \neq \widehat{S}\left[h\left(t_{0}\right)\right],
\end{aligned}
$$

where, due to (59) with $\beta=\xi$ and $r=1$, one has:

$$
\begin{aligned}
& \widehat{\Omega}[\widehat{S}(v), v \mid z, \vartheta]=\frac{1}{v-\vartheta} \exp \left\{-\frac{\widehat{S}(v)+z}{v-\vartheta}\right\}\left[\frac{\widehat{S}(v)}{z}\right]^{(\xi-1) / 2} \\
& \quad \times\left\{\frac{1}{2}\left[\widehat{S}^{\prime}(v)-\frac{2 \widehat{S}(v)}{v-\vartheta}+\xi-\frac{1}{2}\right] I_{\tilde{\zeta}-1}\left[\frac{2 \sqrt{z \widehat{S}(v)}}{v-\vartheta}\right]+\frac{\sqrt{z \widehat{S}(v)}}{v-\vartheta} I_{\tilde{\zeta}}\left[\frac{2 \sqrt{z \widehat{S}(v)}}{v-\vartheta}\right]\right\} .
\end{aligned}
$$

Multiplying both-sides of Equation (91) by $d h(t) / d t$, performing the transformation $\vartheta=h(u)$ in the integral and recalling (79), we obtain the integral Equation (57) with

$$
\begin{aligned}
& \Omega\left[S(t), t \mid x_{0}, t_{0}\right]=\frac{d h(t)}{d t} \widehat{\Omega}\left\{\widehat{S}[h(t)], h(t) \mid \widehat{x}_{0}, h\left(t_{0}\right)\right\}, \\
& \Omega[S(t), t \mid S(u), u]=\frac{d h(t)}{d t} \widehat{\Omega}\{\widehat{S}[h(t)], h(t) \mid \widehat{S}[h(u)], h(u)\}, \quad t_{0}<u<t .
\end{aligned}
$$

Then, (90) follows from (93), making use of (74) and (92).

Let $0 \leq x_{0}<S\left(t_{0}\right)$. We focus on the asymptotic behavior of the FPT pdf of the Feller-type diffusion process (69), with a zero-flux condition in the zero state, through the asymptotically constant boundary (61), with $S(t)>0$, where $\eta(t) \in C^{1}\left[t_{0},+\infty\right)$ is a bounded function, that does not depend on $S$, such that (62) holds. We assume that

$$
\lim _{t \rightarrow+\infty} \alpha(t)=\alpha<0, \quad \lim _{t \rightarrow+\infty} r(t)=r>0,
$$

so that the process allows a steady-state density. Under such assumptions, from (90), one has:

$$
\zeta(S)=-2 \lim _{t \rightarrow+\infty} \Omega\left[S(t), t \mid x_{0}, t_{0}\right]=\frac{|\alpha| S-(\xi-1 / 2) r}{S \Gamma(\xi)}\left(\frac{|\alpha| S}{r}\right)^{\xi} \exp \left\{-\frac{|\alpha| S}{r}\right\} .
$$


Finally, by virtue of (57), for $S \rightarrow+\infty$ and for long periods, the FPT density through the asymptotically constant boundary (61) of the time-inhomogeneous Feller-type process (69) exhibits the following exponential behavior:

$$
g\left[S(t), t \mid x_{0}, t_{0}\right] \simeq \zeta(S) e^{-\zeta(S)\left(t-t_{0}\right)}, \quad S>\frac{(\xi-1 / 2) r}{|\alpha|} .
$$

\section{Conclusions}

In this paper, we have considered the first-passage time problem for a Feller-type diffusion process, having infinitesimal drift $B_{1}(x, t)=\alpha(t) x+\beta(t)$ and infinitesimal variance $B_{2}(x, t)=2 r(t) x$, defined in $[0,+\infty)$, with $\alpha(t) \in \mathbb{R}, \beta(t)>0, r(t)>0$ continuous functions. In Section 2, for the time-homogeneous process, we have determined the Laplace transform of the downwards and upwards FPT densities. In Propositions 1 and 2, some connections between the FPT densities for the Feller and the Wiener processes $(\alpha=0)$ have been discussed, whereas in Propositions 3 and 4 we have analyzed some relations between the FPT densities for Feller and Ornstein-Uhlenbeck processes $(\alpha \neq 0)$. Furthermore, in Section 3, the FPT moments have been investigated by using the Siegert formula. In Section 4 , for $\alpha<0$, the asymptotic behavior of the FPT density through a time-dependent boundary has been discussed for an asymptotically constant boundary and for an asymptotically periodic boundary. Furthermore, the first three moments of FPT density through a constant boundary have been compared with the corresponding asymptotic approximations. Section 5 is dedicated to a time inhomogeneous Feller-type diffusion process with $\beta(t)=\xi r(t)$, for $\xi>0$. In Propositions 6 and 7, the FPT density has been obtained for an exponential time-varying boundary. The FPT densities have been plotted for periodic noise, showing the presence of damped oscillations having the same periodicity as the noise intensity. In Section 6, a second-kind Volterra integral equation was derived for the FPT density of a time-inhomogeneous Feller-type process through a general time-dependent boundary. Finally, such an equation has been used to derive the asymptotic exponential trend of the FPT pdf through an asymptotically constant boundary.

Analytical, asymptotic and computational methods for the evaluation of FPT densities through time-varying boundaries for more general time-inhomogeneous diffusion processes will be the object of future research focused also on contexts of statistical inference.

Author Contributions: Conceptualization, V.G. and A.G.N.; methodology, V.G. and A.G.N.; software, V.G. and A.G.N.; validation, V.G. and A.G.N.; formal analysis, V.G. and A.G.N.; investigation, V.G. and A.G.N.; resources, V.G. and A.G.N.; data curation, V.G. and A.G.N.; visualization, V.G. and A.G.N.; supervision, V.G. and A.G.N. Both authors have read and agreed to the published version of the manuscript.

Funding: This research is partially supported by MIUR_PRIN 2017, Project "Stochastic Models for Complex Systems" and by the Ministerio de Economía, Industria y Competitividad, Spain, under Grant MTM2017-85568-P. This research received no external funding.

Institutional Review Board Statement: Not applicable.

Informed Consent Statement: Not applicable.

Data Availability Statement: Not applicable.

Acknowledgments: The authors are members of the research group GNCS of INdAM.

Conflicts of Interest: The authors declare no conflict of interest.

\section{References}

1. Darling, D.A.; Siegert, A.J.F. The first passage problem for a continuous Markov process. Ann. Math. Stat. 1953, 24, 624-639. [CrossRef]

2. Blake, I.F.; Lindsey, W.C. Level-Crossing Problems for Random Processes. IEEE Trans. Inf. Theory 1973, 19, 295-315. [CrossRef]

3. Giorno, V.; Nobile, A.G.; Ricciardi, L.M. On the densities of certain bounded diffusion processes. Ric. Mat. 2011, 60, 89-124. [CrossRef] 
4. Di Crescenzo, A.; Giorno, V.; Nobile, A.G.; Ricciardi, L.M. On first-passage-time and transition densities for strongly symmetric diffusion processes. Nagoya Math. J. 1997, 145, 143-161. [CrossRef]

5. Gutiérrez, R.; Gonzalez, A.J.; Román, P. Construction of first-passage-time densities for a diffusion process which is not necessarily time-homogeneous. J. Appl. Probab. 1991, 28, 903-909.

6. Di Crescenzo, A; Giorno, V.; Nobile, A.G. Analysis of reflected diffusions via an exponential time-based transformation. J. Stat. Phys. 2016, 163, 1425-1453. [CrossRef]

7. Giorno, V.; Nobile, A.G. On the construction of a special class of time-inhomogeneous diffusion processes. J. Stat. Phys. 2019, 177, 299-323. [CrossRef]

8. Buonocore, A.; Nobile, A.G.; Ricciardi, L.M. A new integral equation for the evaluation of first-passage-time probability densities. Adv. Appl. Probab. 1987, 19, 784-800. [CrossRef]

9. Gutiérrez, R.; Ricciardi, L.M.; Román, P.; Torrez, F. First-passage-time densities for time-non-homogeneous diffusion processes. J. Appl. Probab. 1997, 34, 623-631. [CrossRef]

10. Di Nardo, E.; Nobile, A.G.; Pirozzi, E.; Ricciardi, L.M. A computational approach to first-passage-time problems for Gauss-Markov processes. Adv. Appl. Probab. 2001, 33, 453-482. [CrossRef]

11. Nobile, A.G.; Ricciardi, L.M.; Sacerdote, L. Exponential trends of first passage time densities for a class of diffusion processes with steady-state distribution. J. Appl. Probab. 1985, 22, 611-618. [CrossRef]

12. Nobile, A.G.; Pirozzi, E.; Ricciardi, L.M. Asymptotics and evaluations of FPT densities through varying boundaries for GaussMarkov processes. Sci. Math. Jpn. 2008, 67, 241-266.

13. Herrmann, S.; Zucca, C. Exact simulation of first exit times for one.dimensional diffusion processes. ESAIM Math. Model. Numer. Anal. 2020, 54, 811-844. [CrossRef]

14. Giraudo, M.T.; Sacerdote, L.; Zucca, C. A Monte Carlo method for the simulation of first passage times of diffusion processes. Methodol. Comput. Appl. Probab. 2001, 3, 215-231. [CrossRef]

15. Taillefumier, T.; Magnasco, M. A fast algorithm for the first-passage times of Gauss-Markov processes with Hölder continuous boundaries. J. Stat. Phys. 2010, 140, 1130-1156. [CrossRef]

16. Giorno, V.; Nobile, A.G. On the simulation of a special class of time-inhomogeneous diffusion processes. Mathematics 2021, 9, 818. [CrossRef]

17. Naouara, N.J.B.; Trabelsi, F. Boundary classification and simulation of one-dimensional diffusion processes. Int. J. Math. Oper. Res. 2017, 11, 107-138 [CrossRef]

18. Feller, W. Diffusion processes in genetics. In Proceedings of the Second Berkeley Symposium on Mathematical Statistics and Probability, Berkeley, CA, USA, 31 July-12 August 1950; Statistical Laboratory of the University of California: Berkeley, CA, USA, 1950; pp. 227-246.

19. Lavigne, F.; Roques, L. Extinction times of an inhomogeneous Feller diffusion process: A PDF approach. Expo. Math. 2021, 39, 137-142. [CrossRef]

20. Masoliver, J. Nonstationary Feller process with time-varying coefficients. Phys. Rev. E 2016, 93, 012122. [CrossRef]

21. Pugliese, A.; Milner, F. A structured population model with diffusion in structure space. J. Math. Biol. 2018, 77, 2079-2102. [CrossRef]

22. Di Crescenzo, A.; Nobile, A.G. Diffusion approximation to a queueing system with time-dependent arrival and service rates. Queueing Syst. 1995, 19, 41-62. [CrossRef]

23. Giorno, V.; Lánský, P.; Nobile, A.G.; Ricciardi, L.M. Diffusion approximation and first-passage-time problem for a model neuron. III. A birth-and-death process approach. Biol. Cyber. 1988, 58, 387-404. [CrossRef]

24. Buonocore, A.; Giorno, V.; Nobile, A.G.; Ricciardi, L.M. A neuronal modeling paradigm in the presence of refractoriness. BioSystems 2002, 67, 35-43. [CrossRef]

25. Ditlevsen, S.; Lánský, P. Estimation of the input parameters in the Feller neuronal model. Phys. Rev. E 2006, 73, 061910. [CrossRef]

26. Lánský, P.; Sacerdote, L.; Tomassetti, F. On the comparison of Feller and Ornstein-Uhlenbeck models for neural activity. Biol. Cybern. 1995, 73, 457-465. [CrossRef]

27. Nobile, A.G.; Pirozzi, E. On time non-homogeneous Feller-type diffusion process in neuronal modeling. In Computer Aided Systems Theory_Eurocast 2015, LNCS; Moreno-Díaz, R., Pichler, F., Eds.; Springer International Publishing Switzerland: Cham, Switzerland, 2015; Volume 9520, pp. 183-191.

28. D'Onofrio, G.; Lánský, P.; Pirozzi, E. On two diffusion neuronal models with multiplicative noise: the mean first-passage time properties. Chaos 2018, 28, 043103. [CrossRef]

29. Cox, J.C.; Ingersoll, J.E., Jr.; Ross, S.A. A theory of the term structure of interest rates. Econometrica 1985, 53, 385-407. [CrossRef]

30. Tian, Y.; Zhang, H. Skew CIR process, conditional characteristic function, moments and bond pricing. Appl. Math. Comput. 2018, 329, 230-238. [CrossRef]

31. Maghsoodi, Y. Solution of the extended CIR term structure and bond option valuation. Math. Financ. 1996, 6, 89-109. [CrossRef]

32. Peng, Q.; Schellhorn, H. On the distribution of extended CIR model. Stat. Probab. Lett. 2018, 142, 23-29. [CrossRef]

33. Giorno, V.; Nobile, A.G. Time-inhomogeneous Feller-type diffusion process in population dynamics. Mathematics 2021, 9, 1879. [CrossRef]

34. Ditlevsen, S.; Ditlevsen, O. Parameter estimation from observations of first-passage times of the Ornstein-Uhlenbeck process and the Feller process. Probabilistic Eng. Mech. 2008, 23, 170-179. [CrossRef] 
35. Junginger, A.; Craven, G.T.; Bartsch, T.; Revuelta, F.; Borondo, F.; Benito, R.M.; Hernandez, R. Transition state geometry of driven chemical reactions on time-dependent double-well potentials. Phys. Chem. Chem. Phys. 2016, 18, 30270-30281. [CrossRef]

36. Fortet, R. Les fonctions aléatoires du type de Markoff associées à certaines équations lineàires aux dérivées partielles du type parabolique. J. Math. Pures Appl. 1943, 22, 177-243.

37. Giorno, V.; Nobile, A.G.; Ricciardi, L.M.; Sacerdote, L. Some remarks on the Rayleigh process. J. Appl. Probab. 1986, 23, 398-408. [CrossRef]

38. Linetsky, V. Computing hitting time densities for CIR and OU diffusions. Applications to mean-reverting models. J. Comput. Finance 2004, 7, 1-22. [CrossRef]

39. Masoliver, J.; Perelló, J. First-passage and escape problems in the Feller process. Phys. Rev. E 2012, 86, 041116. [CrossRef] [PubMed]

40. Masoliver, J. Extreme values and the level-crossing problem: An application to the Feller process. Phys. Rev. E 2014, 89, 042106. [CrossRef] [PubMed]

41. Chou, C.-S.; Lin, H.-J. Some Properties of CIR Processes. Stoch. Anal. Appl. 2006, 24, 901-912.

42. Di Nardo, E.; D'Onofrio, G. A cumulant approach for the first-passage-time problem of the Feller square-root process. Appl. Math. Comput. 2021, 391, 125707.

43. Giorno, V.; Nobile, A.G. Time-inhomogeneous Feller-type diffusion process with absorbing boundary condition. J. Stat. Phys. 2021, 183, 1-27. [CrossRef]

44. Feller, W. Two singular diffusion problems. Ann. Math. 1951, 54, 173-182. [CrossRef]

45. Karlin, S.; Taylor, H.W. A Second Course in Stochastic Processes; Academic Press: New York, NY, USA, 1981.

46. Sacerdote, L. On the solution of the Fokker-Planck equation for a Feller process. Adv. Appl. Probab. 1990, 22, 101-110. [CrossRef]

47. Gradshteyn, I.S.; Ryzhik, I.M. Table of Integrals, Series and Products; Academic Press Inc.: Cambridge, MA, USA, 2014.

48. Tricomi, F.G. Funzioni Ipergeometriche Confluenti. Monografie Matematiche a Cura del Consiglio Nazionale delle Ricerche; Edizioni Cremonese: Roma, Italy, 1954.

49. Erdèlyi, A.; Magnus, W.; Oberthettinger, F.; Tricomi, F.G. Tables of Integral Transforms; Mc Graw-Hill: New York, NY, USA, 1954; Volume 1.

50. Abramowitz, I.A.; Stegun, M. Handbook of Mathematical Functions; Dover Publications Inc.: New York, NY, USA, 1972.

51. Spiegel, M.R.; Lipschutz, S.; Liu, J. Mathematical Handbook of Formulas and Tables; Mc Graw Hill: New York, NY, USA, 2009.

52. Siegert, A.J.F. On the first passage time probability problem. Phys. Rev. 1951, 81, 617-623. [CrossRef]

53. Capocelli, R.M.; Ricciardi, L.M. On the transformation of diffusion processes into the Feller process. Math. Biosci. 1976, 29, 219-234. [CrossRef] 